\title{
Pacific
}

Journal of

Mathematics

\section{INTEGRATION OF COUPLING DIRAC STRUCTURES}

OLIVIER BRAHIC AND RUI LOJA Fernandes 


\title{
INTEGRATION OF COUPLING DIRAC STRUCTURES
}

\author{
Olivier BRAHiC AND Rui Loja Fernandes
}

Coupling Dirac structures are Dirac structures defined on the total space of a fibration, generalizing hamiltonian fibrations from symplectic geometry, where one replaces the symplectic structure on the fibers by a Poisson structure. We study the associated Poisson gauge theory, in order to describe the presymplectic groupoid integrating coupling Dirac structures. We find the obstructions to integrability, and we give explicit geometric descriptions of the integration.

1. Introduction 325

2. Coupling Dirac structures 328

3. Integration of coupling Dirac structures I 336

4. Integration of the Yang-Mills-Higgs phase space 341

5. Integration of coupling Dirac structures II 351

Appendix $\quad 360$

References $\quad 366$

\section{Introduction}

A Dirac structure on a manifold $M$ is a (possibly singular) foliation of $M$ by presymplectic leaves. It is well known that Dirac structures can be expressed in terms of a Lagrangian subbundle $L$ of the generalized tangent bundle $T M \oplus T^{*} M$. The bundle $L$ inherits a Lie algebroid structure from the Courant bracket [1990], so Dirac structures are infinitesimal objects. Bursztyn et al. [2004] showed that the global object underlying a given Dirac structure $L$ is a presymplectic groupoid, i.e., a Lie groupoid $\mathcal{G} \rightrightarrows M$ with a multiplicative closed 2-form $\Omega_{\mathcal{G}}$ satisfying a certain nondegeneracy condition. Not all Lie algebroids can be integrated to Lie groupoids, and Dirac structures are no exception: not all Dirac structures can be integrated to presymplectic groupoids. The obstructions to integrability follow from the general obstruction theory discovered by Crainic and Fernandes [2003; 2004].

Brahic was partially supported by CNPq grant 401253/2012-0. Fernandes was partially supported by NSF grants DMS 13-08472 and DMS 14-05671. Both authors acknowledge the support of the Ciências sem Fronteiras program.

MSC2010: 53D17, 58H05.

Keywords: Dirac structure, coupling, presymplectic integration. 
The general methods presented in [Crainic and Fernandes 2003; 2004] allow one to decide if a given Lie algebroid is integrable or not, and to produce a canonical integration in terms of an abstract path space construction. While the obstructions to integrability can be computed explicitly in many examples, describing the canonical integration $\mathcal{G}(L) \rightrightarrows M$ of a given integrable Dirac structure $(M, L)$ is, in general, a very difficult task. However, for a few classes of Dirac structures one does have explicit integrations and often in such cases the construction of the groupoid has a nice geometric flavor.

In this paper we discuss the integration of coupling Dirac structures. The simplest examples of such couplings arise in the context of a symplectic fibration $p: E \rightarrow B$ : a coupling form is a closed 2-form $\omega \in \Omega^{2}(E)$ on the total space of the fibration whose pullback to each fiber $F_{b}$ is the symplectic form $\omega_{F_{b}}$ on the fiber. The obstructions to the existence of such a coupling form are well known and we will recall them below. We are interested in the more general situation of a Poisson fibration: now one looks for a coupling Dirac structure on the total space of the fibration which glues the Poisson structures on the fibers. This idea of a coupling is only a rough approximation: Dirac structures are very flexible and extra care must be taken in defining precisely the notion of a coupling [Dufour and Wade 2008; Brahic and Fernandes 2008; Vaisman 2006; Wade 2008].

Coupling Dirac structures appear very naturally in Poisson and Dirac geometry. One reason is that tubular neighborhoods of symplectic and presymplectic leaves in arbitrary Poisson and Dirac manifolds are always coupling Dirac structures. Our first main result concerning the integration of couplings can be stated as follows:

Theorem 1.1. Let $L$ be a coupling Dirac structure on $p: E \rightarrow B$. If $L$ is integrable and $(\mathcal{G}, \Omega) \rightrightarrows B$ is a source connected, presymplectic groupoid integrating $L$, then $\Omega$ is a coupling form for a fibration $\bar{p}: \mathcal{G} \rightarrow \Pi(B)$ obtained by integrating the algebroid morphism $p_{*} \circ \sharp: L \rightarrow T B$.

In other words, coupling Dirac structures integrate to coupling forms. Moreover, one can express the geometric data of the integration in terms of the geometric data associated with the coupling Dirac structure $L$. As a consequence of this result, any presymplectic groupoid integrating a coupling Dirac structure on $p: E \rightarrow B$ is Morita equivalent to a symplectic groupoid integrating the induced vertical Poisson structure on a fiber $E_{b}$.

The previous result describes the symplectic geometry of the integration. One is also interested in the groupoid structure of the integration and the obstructions to integrability. Our inspiration to deal with this integration problem comes from a beautiful gauge construction, known as the Yang-Mills setup, which yields coupling Dirac structures [Brahic and Fernandes 2014; Guillemin et al. 1996; McDuff and Salamon 1998; Weinstein 1980; Wade 2008]. 
Start with a principal $G$-bundle $P \rightarrow B$, with a connection $\Gamma$, and a hamiltonian action $G \times F \rightarrow F$ on a Poisson manifold $\left(F, \pi_{F}\right)$, and construct a coupling Dirac structure $L$ on the associated bundle $E=P \times_{G} F$ extending the Poisson structures on the fibers. This construction can be further twisted by a closed 2-form on the base $B$, and it leads to many examples of coupling Dirac structures.

We show that one can integrate a Yang-Mills phase space as follows:

(i) First integrate the fiber $\left(F, \pi_{F}\right)$ to a symplectic groupoid $\mathcal{F} \rightrightarrows F$, and the principal $G$-bundle $P \rightarrow B$ to the gauge groupoid $\mathcal{G}(P) \rightrightarrows B$.

(ii) Then integrate the vertical Poisson structure Ver* to a fibered symplectic groupoid $\mathcal{G}_{V}=P \times{ }_{G} \mathcal{F} \rightrightarrows E$.

(iii) The gauge groupoid $\mathcal{G}(P) \rightrightarrows B$ acts on the fibered groupoid $\mathcal{G}_{V} \rightrightarrows E \rightarrow B$, yielding a semidirect product groupoid $\mathcal{G}(P) \ltimes \mathcal{G}_{V} \rightrightarrows E$.

(iv) Finally, the integration of the Yang-Mills phase space is a quotient

$$
\mathcal{G}(L)=\mathcal{G}(P) \ltimes \mathcal{G}_{V} / \mathcal{C},
$$

where $\mathcal{C}$ is a certain curvature groupoid.

Along the way we obtain the obstructions to integrability of a Yang-Mills phase space. Our integration procedure does not uses the principal bundle connection; hence, all the different couplings obtained by varying the connection have the same integrating Lie groupoid $\mathcal{G} \rightrightarrows E$. On the other hand, we also provide a construction for the presymplectic form $\Omega_{\mathcal{G}}$, which obviously depends on the choice of principal connection.

We show that, provided one is willing to accept infinite dimensional principal bundles, every coupling on a locally trivial fibration arises as a Yang-Mills phase space. This observation turns out to be the clue to integrate arbitrary coupling Dirac structures:

Theorem 1.2. Let $L$ be a coupling Dirac structure on $E \rightarrow B$. The source 1-connected groupoid $\mathcal{G}(L)$ integrating $L$ naturally identifies with equivalence classes in $P(T B) \ltimes_{B} \mathcal{G}$ (Ver*) under the equivalence relation:

- $\left(\gamma_{0}, g_{0}\right) \sim\left(\gamma_{1}, g_{1}\right)$ if and only if there exists a homotopy $\gamma_{B}: I \times I \rightarrow B$, $(t, \epsilon) \mapsto \gamma_{B}^{\epsilon}(t)$ between $\gamma_{0}$ and $\gamma_{1}$, such that $g_{1}=\partial\left(\gamma_{B}, t\left(g_{0}\right)\right) \cdot g_{0}$.

where $\partial: P(T B) \times{ }_{B} E \rightarrow \mathcal{G}\left(\right.$ Ver* $\left.^{*}\right)$ is a certain "groupoid" homomorphism that can be computed explicitly.

The quotes in "groupoid" are used here because the path space $P(T B)$ is not really a groupoid, since associativity only holds up to isomorphism.

Again, Theorem 1.2 should be viewed as an infinite dimensional version of the groupoid integrating the Yang-Mills phase space. It also gives rise to the 
integrability obstructions of coupling Dirac structure. Namely, one checks that the restriction of the map $\partial: P(T B) \times_{B} E \rightarrow \mathcal{G}\left(\operatorname{Ver}^{*}\right)$ to a sphere in $B$ based at some $b \in B$ (seen as a map $\gamma_{B}: I^{2} \rightarrow B$ such that $\gamma_{B}\left(\partial I^{2}\right)=\{b\}$ ) is independent of its homotopy class. Then, if we let $\mathcal{M}:=\partial\left(\pi_{2}(B) \times{ }_{B} E\right)$, which we call the monodromy groupoid of the fibration, then we have the theorem:

Theorem 1.3. Let $L$ be a coupling Dirac structure on $E \rightarrow B$ and assume that the associated connection $\Gamma$ is complete. Then, $L$ is an integrable Lie algebroid if and only if the following conditions hold:

(i) the typical Poisson fiber $\left(E_{x},\left.\pi_{V}\right|_{E_{x}}\right)$ is integrable;

(ii) the injection $\mathcal{M} \hookrightarrow \mathcal{G}$ (Ver*) is an embedding.

The transgression map $\partial: \pi_{2}(B) \times_{B} E \rightarrow \mathcal{G}$ (Ver*) is computable in many examples, and so are the integrability obstructions of Theorem 1.3. We refer to the last section of the paper, where we will discuss for example the trivial fibration $p: \mathbb{S}^{2} \times \mathfrak{s o}^{*}(3) \rightarrow \mathbb{S}^{2}$, with the usual Lie-Poisson structure on the fibers. Using Theorem 1.3 one can see that there is only a 2-parameter family of integrable Dirac couplings of rank 4, while there is an infinite dimensional family of nonintegrable Dirac couplings of rank 4.

\section{Coupling Dirac structures}

The notion of a coupling was first introduced in the context of Dirac geometry [Vaisman 2006] (see also [Brahic and Fernandes 2008; Dufour and Wade 2008; Wade 2008]) but their origins lie in the theory of symplectic and hamiltonian fibrations; see, e.g., [Guillemin et al. 1996; McDuff and Salamon 1998]. In this section we recall the definition of a coupling Dirac structure and study its first properties.

2A. Fiber nondegenerate Dirac structures. We shall use some standard notions from Dirac geometry; see, e.g., [Courant 1990]. So given a smooth manifold $M$, we denote by $\mathbb{T} M:=T M \oplus T^{*} M$ its generalized tangent bundle. The space of sections $\Gamma(\mathbb{T} M)$ has two natural pairings:

$$
\langle(X, \alpha),(Y, \beta)\rangle_{ \pm}:=\frac{1}{2}\left(i_{Y} \alpha \pm i_{X} \beta\right),
$$

and a skew-symmetric bracket, called the Courant bracket, given by

$$
\llbracket(X, \alpha),(Y, \beta) \rrbracket:=\left([X, Y], \mathcal{L}_{X} \beta-\mathcal{L}_{Y} \alpha+\mathrm{d}\langle(X, \alpha),(Y, \beta)\rangle_{-}\right) .
$$

An almost Dirac structure $L$ on $M$ is a subbundle $L \subset \mathbb{} M:=T M \oplus T^{*} M$ of the generalized tangent bundle, which is maximally isotropic with respect to $\langle\cdot, \cdot\rangle_{+}$. An almost Dirac structure is said to be a Dirac structure if it is furthermore closed under the bracket $\llbracket \cdot, \cdot \rrbracket$. 
In general, the Courant bracket does not satisfy the Jacobi identity. For a Dirac structure $L$, however, its restriction to $\Gamma(L)$ yields a Lie bracket, and if we let $\sharp: L \rightarrow T M$ be the restriction of the projection to $T M$, then $(L, \mathbb{[} \cdot, \cdot \mathbb{\rrbracket}, \sharp)$ defines a Lie algebroid. Each leaf of the corresponding characteristic foliation, obtained by integrating the singular distribution $\operatorname{Im} \sharp$, carries a presymplectic form $\omega$ : if $X, Y \in \operatorname{Im} \sharp$, we can choose $\alpha, \beta \in T^{*} M$ such that $(X, \alpha),(Y, \beta) \in L$ and set

$$
\omega(X, Y):=\langle(X, \alpha),(Y, \beta)\rangle_{-}=i_{Y} \alpha=-i_{X} \beta .
$$

One can check that this definition is independent of choices and that $\omega$ is indeed closed. Thus we may think of a Dirac manifold as a manifold foliated by (possibly singular) presymplectic leaves.

In what follows, unless otherwise stated, by a fibration $p: E \rightarrow B$ we mean a surjective submersion.

Definition 2.1. Let $p: E \rightarrow B$ be a fibration. An almost Dirac structure $L$ on $E$ is called fiber nondegenerate if

$$
\left(\operatorname{Ver} \oplus \operatorname{Ver}^{0}\right) \cap L=\{0\} .
$$

Here, Ver $:=\operatorname{ker} p_{*} \subset T E$ denotes the vertical distribution, and $\operatorname{Ver}^{0} \subset T^{*} E$ its annihilator. When $L$ is both Dirac and fiber nondegenerate, we shall refer to $L$ as a coupling Dirac structure.

In the terminology of [Mărcuţ and Frejlich 2013], when $L$ is a Poisson structure, this condition means that the fibers of $p: E \rightarrow B$ are Poisson transversals.

In order to understand the geometric meaning of this definition, one needs to decompose a fiber nondegenerate almost structure $L$ into its various components:

- First, $L$ gives rise to an Ehresmann connection by setting:

$$
\text { Hor }:=\left\{X \in T E: \exists \alpha \in(\text { Ver })^{0} \text { such that }(X, \alpha) \in L\right\} .
$$

The fact that Hor $\oplus \operatorname{Ver}=T E$ is an easy consequence of (4).

- Next, it follows from (5) that the horizontal distribution Hor is contained in the characteristic distribution of $L$. Hence, we obtain a horizontal 2-form $\omega_{H} \in \Omega^{2}$ (Hor) by restricting the natural 2-form on the characteristic distribution to Hor. More precisely, (4) and (5) together show that for each $X \in$ Hor, there exists a unique $\alpha \in \operatorname{Ver}^{0}$ such that $(X, \alpha) \in L$. The skew-symmetric bilinear form $\omega_{H}:$ Hor $\times$ Hor $\rightarrow \mathbb{R}$ is defined by

$$
\omega_{H}\left(X_{1}, X_{2}\right):=\left\langle\left(X_{1}, \alpha_{1}\right),\left(X_{2}, \alpha_{2}\right)\right\rangle_{-},
$$

where $\alpha_{1}, \alpha_{2} \in \operatorname{Ver}^{0}$ are the unique elements with $\left(X_{1}, \alpha_{1}\right),\left(X_{2}, \alpha_{2}\right) \in L$. Since $L$ is maximal isotropic, this 2 -form can also be written $\omega_{H}\left(X_{1}, X_{2}\right)=$ $\alpha_{1}\left(X_{2}\right)=-\alpha_{2}\left(X_{1}\right)$. 
- Finally, we can associate to $L$ a vertical bivector field $\pi_{V} \in \mathfrak{X}^{2}($ Ver). To see this, first observe that the annihilator of the horizontal distribution is:

$$
\operatorname{Hor}^{0}=\left\{\alpha \in T^{*} E: \exists X \in \text { Ver such that }(X, \alpha) \in L\right\} .
$$

This, together with (4), shows that for each $\alpha \in \operatorname{Hor}^{0}$, there exists a unique $X \in$ Ver such that $(X, \alpha) \in L$. Then one can define a skew-symmetric bilinear form $\pi_{V}: \operatorname{Hor}^{0} \times \operatorname{Hor}^{0} \rightarrow \mathbb{R}$ by letting:

$$
\pi_{V}\left(\alpha_{1}, \alpha_{2}\right):=\left\langle\left(X_{1}, \alpha_{1}\right),\left(X_{2}, \alpha_{2}\right)\right\rangle_{-},
$$

where $X_{1}, X_{2} \in$ Ver are the unique elements with $\left(X_{1}, \alpha_{1}\right),\left(X_{2}, \alpha_{2}\right) \in L$. Since $L$ is maximal isotropic, the form $\pi_{V}: \operatorname{Hor}^{0} \times \operatorname{Hor}^{0} \rightarrow \mathbb{R}$ can also be written as $\pi_{V}\left(\alpha_{1}, \alpha_{2}\right)=\alpha_{1}\left(X_{2}\right)=-\alpha_{2}\left(X_{1}\right)$. Notice that the splitting $T E=\operatorname{Hor} \oplus \operatorname{Ver}$ allows us to identify $\operatorname{Hor}^{0}=$ Ver*; thus, $\pi_{V}$ becomes a bivector field on the fibers of $p: E \rightarrow B$.

A more geometric interpretation of $\pi_{V}$ is that it is formed by the pullback to each fiber of the Dirac structure $L$; an easy computation shows that for each fiber $F_{b}=p^{-1}(b)$, the pullback Dirac structure $i_{b}^{*} L$ under the inclusion $i_{b}: F_{b} \hookrightarrow E$ coincides with $\pi_{V}$ :

$$
i_{b}^{*} L:=\left\{\left(X,\left.\alpha\right|_{\text {Ver }}\right) \in \operatorname{Ver} \oplus \operatorname{Ver}^{*}:(X, \alpha) \in L\right\}=\operatorname{Graph}\left(\pi_{V}\right) .
$$

The preceding discussion justifies the following definition:

Definition 2.2. A geometric data on a fibration $p: E \rightarrow B$ is a triple $\left(\pi_{V}, \Gamma, \omega_{H}\right)$, where

- $\pi_{V} \in \mathfrak{X}^{2}$ (Ver) is a vertical bivector field.

- $\Gamma$ is an Ehresmann connection, whose horizontal distribution is denoted Hor,

- $\omega_{H} \in \Omega^{2}$ (Hor) is a horizontal 2-form,

Proposition 2.3. Given a fibration $E \rightarrow B$, there is a one-to-one correspondence between fiber nondegenerate almost Dirac structures and geometric data on the fibration.

Proof. We have seen above how to associate to a fiber nondegenerate almost Dirac structure $L$, a geometric data $\left(\pi_{V}, \Gamma, \omega_{H}\right)$. Conversely, given a geometric data $\left(\Gamma_{L}, \omega_{H}, \pi_{V}\right)$ on a fibration $p: E \rightarrow B$, define an almost Dirac structure $L$ by

$$
L:=\left\{\left(X+\pi_{V}^{\sharp}(\alpha), i_{X} \omega_{H}+\alpha\right): X \in \text { Hor and } \alpha \in \text { Hor }^{0}\right\} .
$$

Notice that by using the identifications $\operatorname{Hor}^{0}=\operatorname{Ver}^{*}$ and Ver ${ }^{0}=$ Hor* $^{*}$, we obtain simply $L=$ Graph $\omega_{H} \oplus$ Graph $\pi_{V}$, which will prove to be a meaningful way of presenting $L$ later on. 
Given a fiber nondegenerate almost Dirac structure $L$ with associated geometric data $\left(\Gamma, \omega_{H}, \pi_{V}\right)$, we now express the conditions on this data which will guarantee that $L$ is a Dirac structure, i.e., that it is closed under the Courant bracket.

Let us first introduce some notations associated with the connection $\Gamma$. For a vector field $v \in \mathfrak{X}(B)$, we denote by $h(v) \in \mathfrak{X}(E)$ its horizontal lift. The exterior covariant differential $\mathrm{d}_{\Gamma}: \Omega^{k}(B) \otimes C^{\infty}(E) \rightarrow \Omega^{k+1}(B) \otimes C^{\infty}(E)$ is given by

$$
\begin{aligned}
\mathrm{d}_{\Gamma} \omega\left(v_{0}, \ldots, v_{k}\right):=\sum_{i=0}^{k}(-1)^{i} & \mathcal{L}_{h\left(v_{i}\right)} \omega\left(v_{0}, \ldots, \hat{v}_{i}, \ldots, v_{k}\right) \\
& +\sum_{i<j}(-1)^{i+j} \omega\left(\left[v_{i}, v_{j}\right], v_{0}, \ldots, \hat{v}_{i}, \ldots, \hat{v}_{j}, \ldots, v_{k}\right)
\end{aligned}
$$

The curvature of $\Gamma$ will be denoted by $\operatorname{Curv}_{\Gamma} \in \Omega^{2}(B$, Ver) and is defined by

$$
\operatorname{Curv}_{\Gamma}(v, w):=[h(v), h(w)]-h([v, w]) \quad \text { for } v, w \in \mathfrak{X}(B) .
$$

The curvature measures the failure of Hor in being involutive or, equivalently, the failure of $\mathrm{d}_{\Gamma}$ being a differential since

$$
\mathrm{d}_{\Gamma}^{2} f(u, v)=\mathcal{L}_{\operatorname{Curv}(u, v)} f,
$$

for any $f \in C^{\infty}(E)$ and $u, v \in \mathfrak{X}(B)$.

Proposition 2.4. Let $\left(\pi_{V}, \Gamma, \omega_{H}\right)$ be the geometric data determined by a fiber nondegenerate almost Dirac structure $L$ on a fiber bundle $p: E \rightarrow B$. Then, $L$ is a Dirac structure if and only if the following conditions hold:

(i) $\pi_{V}$ is a vertical Poisson structure:

$$
\left[\pi_{V}, \pi_{V}\right]=0
$$

(ii) parallel transport along $\Gamma$ preserves the vertical Poisson structure:

$$
\mathcal{L}_{h(v)} \pi_{V}=0, \quad \text { for any } v \in \mathfrak{X}(B) ;
$$

(iii) the horizontal 2-form $\omega_{H}$ is $\Gamma$-closed:

$$
\mathrm{d}_{\Gamma} \omega_{H}=0
$$

(iv) the curvature is hamiltonian:

$$
\operatorname{Curv}(u, v)=\pi_{V}^{\sharp}\left(\mathrm{d} i_{h(u)} i_{h(v)} \omega_{H}\right), \quad \text { for any } u, v \in \mathfrak{X}(B) \text {. }
$$

A proof of Proposition 2.4 can be found in [Brahic and Fernandes 2008]. We shall refer to (9) as the curvature identity. 
2B. Examples. The notion of coupling Dirac structure contains as special cases the notion of coupling form for symplectic fibrations (see, e.g., [Guillemin et al. 1996]) and the coupling Poisson tensor considered by Vorobjev [2001]. We now recall these examples as well as other ones.

2B1. Coupling forms. Let $\omega$ be a closed 2-form on the total space of a fibration $p: E \rightarrow B$. The associated Dirac structure $L:=\operatorname{Graph}(\omega)$ is fiber nondegenerate if and only if the pullback of $\omega$ to each fiber is a nondegenerate 2-form. In this case, the fibration with the restriction of $\omega$ to the fibers becomes a symplectic fibration. The geometric data $\left(\pi_{V}, \Gamma, \omega_{H}\right)$ associated to $L$ has a nondegenerate vertical Poisson structure $\pi_{V}$ which coincides with the inverse of the restriction of $\omega$ to the fibers.

The converse is also true: a fiber nondegenerate Dirac structure $L$ for which the geometric data $\left(\pi_{V}, \Gamma, \omega_{H}\right)$ has a nondegenerate vertical Poisson structure $\pi_{V}$ is determined by a presymplectic form $\omega$. In fact, it follows from (8) that $L$ is the graph of the closed 2-form

$$
\omega=\omega_{H} \oplus\left(\pi_{V}\right)^{-1} .
$$

Hence, fiber nondegenerate presymplectic forms are the same as coupling forms for symplectic fibrations [Guillemin et al. 1996].

2B2. Coupling Poisson structures. Let $\pi$ be a Poisson structure on the total space of a fibration $p: E \rightarrow B$. The Dirac structure $L=\operatorname{Graph}(\pi)$ is fiber nondegenerate if and only if $\pi$ is horizontal nondegenerate in the sense of [Vorobjev 2001], i.e., if the bilinear form $\left.\pi\right|_{\operatorname{Ver}^{0}}: \operatorname{Ver}^{0} \times \operatorname{Ver}^{0} \rightarrow \mathbb{R}$ is nondegenerate. In terms of the associated geometric data $\left(\pi_{V}, \Gamma_{L}, \omega_{H}\right)$ the Poisson structure on the fibers is $\pi_{V}$ and $\omega_{H}$ is nondegenerate; in fact, $\pi$ induces an isomorphism $\operatorname{Ver}^{0} \rightarrow$ Hor under which $\omega_{H}$ coincides with the restriction $\left.\pi\right|_{\operatorname{Ver}^{0}}$.

The converse is also true: a fiber nondegenerate Dirac structure $L$ for which the horizontal 2-form $\omega_{H}$ is nondegenerate is given by a Poisson structure $\pi$. In fact, it follows from (8) that

$$
\pi=\left(\omega_{H}\right)^{-1} \oplus \pi_{V} .
$$

Hence, fiber nondegenerate Poisson structures are the same thing as the horizontal nondegenerate Poisson structures of Vorobjev.

2B3. Neighborhood of a presymplectic leaf. Let $L$ be any Dirac structure on a manifold $M$ and fix a presymplectic leaf $(S, \omega)$ of $L$. Then, the restriction of $L$ to any sufficiently small tubular neighborhood $p: \nu(S) \rightarrow S$ of the leaf is a coupling Dirac structure. To see this, one observes that along $S$ :

$$
L_{x} \cap\left(v(S)_{x} \oplus v_{x}(S)^{0}\right)=\{0\}, \quad \text { for all } x \in S .
$$


It follows that $L$ is fiber nondegenerate on a sufficiently small neighborhood of the zero section.

This shows that in a neighborhood of a presymplectic leaf the Dirac structure takes a special form and we can associate to it the geometric data $\left(\pi_{V}, \Gamma, \omega_{H}\right)$. The Poisson structure $\pi_{V}$ is the transverse Poisson structure along $S$, while $S$ (viewed as the zero section) is an integral leaf of Hor and the 2-form $\omega_{H}$ restricted to this leaf coincides with $\omega$. Note that, in general, the distribution Hor fails to be integrable at other points.

2B4. Reduction of canonical bundles. Let $P \rightarrow M$ be a principal $G$-bundle. The action of $G$ naturally lifts to a hamiltonian action of $G$ on $\left(T^{*} P, \omega_{\text {can }}\right)$. Clearly, $T^{*} P$ is itself a principal $G$-bundle, sometimes called a canonical bundle, and it follows that the base manifold $T^{*} P / G$ has an induced Poisson structure $\pi$.

Each choice of a principal bundle connection $\theta: T P \rightarrow \mathfrak{g}$ induces a projection map $p_{\theta}: T^{*} P / G \rightarrow T^{*} M$. It is easy to check that, for any choice of connection, the Dirac structure $L=\operatorname{Graph}(\pi)$ on $E=T^{*} P / G$ is a coupling Dirac structure over $B=T^{*} M$.

2B5. Yang-Mills-Higgs phase spaces. There is a construction using principal bundles and hamiltonian actions which leads to an important class of coupling Dirac structures.

Definition 2.5. A classical Yang-Mills-Higgs setting is a triple $(P, G, F)$ where $P \rightarrow B$ is a principal $G$-bundle and $\left(F, \pi_{F}\right)$ is a $G$-hamiltonian Poisson manifold with equivariant moment map $\mu_{F}: F \rightarrow \mathfrak{g}^{*}$.

Proposition 2.6. Let $(P, G, F)$ be a classical Yang-Mills-Higgs setting. Each choice of a principal bundle connection $\theta: T P \rightarrow \mathfrak{g}$ determines a coupling Dirac structure on the associated fiber bundle $E:=P \times{ }_{G} F \rightarrow B$.

The construction is well known (see [Brahic and Fernandes 2014; Weinstein 1980; Wade 2008]), so it will only be sketched. First, the connection $\theta: T P \rightarrow \mathfrak{g}$ gives a $G$-equivariant embedding $i_{\theta}:(\operatorname{ker} \mathrm{d} p)^{*} \hookrightarrow T^{*} P$, where $p: P \rightarrow B$ is the principal bundle projection. This allows us to pullback the hamiltonian $G$-space $\left(T^{*} P, \omega_{\text {can }}, \mu_{\text {can }}\right)$, where $\mu_{\text {can }}: T^{*} P \rightarrow \mathfrak{g}^{*}$ is the dual of the infinitesimal action $\mathfrak{g} \rightarrow T P$, to obtain a hamiltonian $G$-space $\left((\operatorname{ker} \mathrm{d} p)^{*}, L_{\theta}, \mu_{\theta}\right)$, where $L_{\theta}:=$ $\operatorname{Graph}\left(i_{\theta}^{*} \omega_{\text {can }}\right)$ and $\mu_{\theta}:(\operatorname{ker} \mathrm{d} p)^{*} \rightarrow \mathfrak{g}^{*}$ is the composition $\mu_{\text {can }} \circ i_{\theta}$.

Next, combine the hamiltonian $G$-spaces $\left((\operatorname{ker} \mathrm{d} p)^{*}, L_{\theta}, \mu_{\theta}\right)$ and $\left(F, L_{\pi_{F}}, \mu_{F}\right)$, where $L_{\pi_{F}}=\operatorname{Graph}\left(\pi_{F}\right)$, to obtain a hamiltonian $G$-space

$$
\left((\operatorname{ker} \mathrm{d} p)^{*} \times F, L_{\theta} \times L_{\pi_{F}}, \mu_{\theta}+\mu_{F}\right),
$$

where $G$ acts diagonally on $(\operatorname{ker} \mathrm{d} p)^{*} \times F$. 
Finally, observe that the hamiltonian quotient

$$
\left((\operatorname{ker} \mathrm{d} p)^{*} \times F\right) / / G:=\left\{(v, f) \in(\operatorname{ker} \mathrm{d} p)^{*} \times F: \mu_{\theta}(u)+\mu_{F}(f)=0\right\} / G
$$

is isomorphic to $E:=P \times_{G} F$ : the map $[(v, f)] \mapsto[(u, f)]$, where $v \in \operatorname{kerd}_{u} p$, gives the desired isomorphism. It follows that $E$ has a quotient Dirac structure $L$, and this is indeed a coupling Dirac structure for the fibration $E \rightarrow B$.

The associated coupling Dirac structure can be described as follows. Since $G$ acts on $F$ by Poisson automorphisms, the associated bundle $E:=P \times{ }_{G} F$ has an induced vertical Poisson structure $\pi_{V}$ with typical fiber $\left(F, \pi_{F}\right)$. The induced connection $\Gamma$ on $E$ is a Poisson connection. Denoting by $\omega_{\theta} \in \Omega^{2}(B, \mathfrak{g})$ the curvature of the principal connection $\theta: T P \rightarrow \mathfrak{g}$, one obtains a well defined horizontal 2-form $\omega_{H}$ on $E$ by setting

$$
\omega_{H}\left(h\left(v_{1}\right), h\left(v_{2}\right)\right):=\left\langle\mu_{F}, \omega_{\theta}\left(v_{1}, v_{2}\right)\right\rangle .
$$

The triple $\left(\pi_{V}, \Gamma, \omega_{H}\right)$ is the geometric data associated with $L$. One can also easily check that this triple satisfies the conditions in proposition 2.4.

Dirac structures obtained in this way are sometimes called classical Yang-MillsHiggs phase spaces. We will be interested in the problem of integrability of such structures. In particular, the integrability of $\left(F, \pi_{F}\right)$ is not enough to ensure the integrability of the associated bundle, as shown in the following example.

Example 2.7. Consider the Hopf fibration $P=\mathbb{S}^{3} \rightarrow \mathbb{S}^{2}$, seen as an $\mathbb{S}^{1}$-principal bundle. One can choose a principal connection $\theta$ whose curvature is given by $\omega_{\theta}=p^{*} \omega$, where $\omega$ is the standard symplectic form on $\mathbb{S}^{2}$. Consider, furthermore, $F=\mathbb{R}$ endowed with the trivial Poisson structure $\pi_{F}=0$, and let $\mathbb{S}^{1}$ act trivially on $F$. Any smooth function $f: F \rightarrow \mathbb{R}$ serves as a momentum map.

The associated bundle is trivial: $E=P \times_{\mathbb{S}^{1}} F=\mathbb{S}^{2} \times \mathbb{R}$. Moreover, it is easily checked that the induced coupling Dirac structure has presymplectic leaves $\left(\mathbb{S}^{2} \times\{x\}, f(x) \omega\right)$. Here, the associated geometric data is given by $\left(\pi_{V}\right.$, Hor, $\left.\omega_{H}\right)$ where $\pi_{V}=0$, Hor is the flat connection given by the trivialization, and $\omega_{H}:=f p^{*} \omega$. Although $\pi_{V}$ is integrable, the coupling Dirac structure $L$ is not integrable whenever $f$ has some critical point; see [Crainic and Fernandes 2004].

Remark 2.8. General coupling Dirac structures can be seen as infinite dimensional Yang-Mills-Higgs phase spaces, provided one allows for infinite dimensional structure groups, as shown in [Brahic and Fernandes 2008]. A precise formulation requires the theory of Fréchet manifolds and Fréchet Lie groups. However, one can use this Poisson gauge theory heuristically, offering guidance on how to extend constructions which work for a Yang-Mills-Higgs phase space to a general coupling Dirac structure. We will use this principle later in our construction of the integration of general coupling Dirac structures. 
2C. Coupling Dirac structures as extensions. In the Yang-Mills-Higgs approach to general coupling Dirac structures, one must allow for infinite dimensional structure groups. An alternative approach (see [Brahic 2010]) is to observe that coupling Dirac structures give rise to Lie algebroid extensions.

Proposition 2.9. Let $L$ be a coupling Dirac structure on $p: E \rightarrow B$. The morphism $p_{*} \circ \sharp: L \rightarrow T B$ induces a Lie algebroid extension

$$
\operatorname{Graph}\left(\pi_{V}\right) \hookrightarrow L \rightarrow T B
$$

Moreover, the decomposition (8) induces an Ehresmann connection with horizontal space $\operatorname{Graph}\left(\omega_{H}\right)$, namely

$$
L=\operatorname{Graph}\left(\pi_{V}\right) \oplus \operatorname{Graph}\left(\omega_{H}\right) .
$$

Proof. The map $p_{*} \circ \sharp: L \rightarrow T B$ is clearly a Lie algebroid morphism, being the composition of algebroid morphisms. It is a surjective morphism because Hor $\subset \operatorname{Im} \sharp$ and, since it covers the surjective submersion $p: E \rightarrow B$, it defines a Lie algebroid extension. The fiber nondegeneracy condition also implies that the kernel of the extension is given by

$$
\operatorname{ker}\left(p_{*} \circ \sharp\right)=\sharp^{-1}(\operatorname{Ver})=\operatorname{Graph}\left(\pi_{V}\right) .
$$

Hence, the kernel is exactly $\operatorname{Graph}\left(\pi_{V}\right) \subset \operatorname{Ver}^{*} \oplus$ Ver. The decomposition (11) gives a complementary vector subbundle to this kernel, i.e., an Ehresmann connection in the sense of Brahic.

For a Lie algebroid extension which is split, as in (11), there is a natural decomposition of its Lie bracket [Brahic 2010, Lemma 1.8].

First, we may identify $\operatorname{Graph}\left(\pi_{V}\right)$ with Ver*, so sections of $\operatorname{Graph}\left(\pi_{V}\right)$ are identified with vertical forms. Vertical forms $\alpha, \beta \in \Gamma\left(\operatorname{Ver}^{*}\right)$ come naturally equipped with a bracket and an anchor inherited from $\pi_{V}$ :

$$
\begin{aligned}
\sharp_{V}(\alpha) & :=\pi_{V}^{\sharp}(\alpha), \\
{[\alpha, \beta]_{V} } & :=\mathcal{L}_{\sharp_{V}}(\alpha) \beta-\mathcal{L}_{\sharp_{V}(\beta)} \alpha-\mathrm{d}_{V} \pi_{V}(\alpha, \beta),
\end{aligned}
$$

where $\mathrm{d}_{V}: C^{\infty}(E) \rightarrow \Gamma\left(\right.$ Ver* $\left.^{*}\right)$ denotes the vertical de Rham differential. Since $\pi_{V}$ is Poisson, this makes Ver* into a Lie algebroid.

Second, for each $v \in \mathfrak{X}(B)$ there is a unique section $h^{*}(v)$ of $\operatorname{Graph}\left(\omega_{H}\right)$ such that $\mathrm{d} p \circ \sharp\left(h^{*}(v)\right)=v$. In fact, we have an isomorphism $\sharp$ : $\operatorname{Graph}\left(\omega_{V}\right) \rightarrow$ Hor, so we can first lift $v$ to $h(v) \in \Gamma$ (Hor) and then apply $\sharp^{-1}$, which gives

$$
h^{*}(v)=\left(h(v), i_{h(v)} \omega_{H}\right) \in \Gamma\left(\operatorname{Graph}\left(\omega_{H}\right)\right) .
$$

We refer to $h^{*}: \mathfrak{X}(B) \rightarrow \Gamma\left(\operatorname{Graph}\left(\omega_{H}\right)\right)$ as the cohorizontal lifting map. 
Sections of $L$ are generated by sections $\alpha \in \Gamma\left(\operatorname{Ver}^{*}\right)$ and $h^{*}(v)$, for $v \in \mathfrak{X}(B)$, so the Lie bracket on $L$ is entirely determined by its value on these two types of sections.

Proposition 2.10 (Splitting Brackets). Let L be a coupling Dirac structure on $E \rightarrow B$. Under the decomposition (11) the Lie bracket of L satisfies:

$$
\begin{gathered}
{[\alpha, \beta]_{L}=[\alpha, \beta]_{V}, \quad\left[h^{*}(v), \alpha\right]_{L}=\mathcal{L}_{h(v)} \alpha,} \\
{\left[h^{*}(v), h^{*}(w)\right]_{L}=h^{*}([v, w])+\mathrm{d}_{V} \omega_{H}(h(v), h(w)),}
\end{gathered}
$$

while the anchor takes the form:

$$
\sharp\left(h^{*}(v)+\alpha\right)=h(v)+\sharp_{V}(\alpha)
$$

for any elements $v, w \in \mathfrak{X}(B)$ and $\alpha, \beta \in \Gamma\left(\right.$ Ver $\left.^{*}\right)$.

Proof. The proposition follows from straightforward computation using (2) and the identifications Hor* $\simeq$ Ver $^{0}$ and Ver $^{*} \simeq$ Hor $^{0}$.

In particular, we see that the curvature of $\operatorname{Graph}\left(\omega_{H}\right)$, as an Ehresmann connection on the Lie algebroid extension $L$, is given by

$$
\left(\pi_{V}^{\sharp} \mathrm{d}_{V} \omega_{H}, \mathrm{~d}_{V} \omega_{H}\right) \in \Omega^{2}\left(B, \Gamma\left(\operatorname{Graph}\left(\pi_{V}\right)\right)\right) .
$$

Notice that this is just another way of expressing the curvature identity (9).

\section{Integration of coupling Dirac structures I}

As stated in the introduction, our main aim is to understand the integration of coupling Dirac structures. We now take care of the symplectic geometry, showing that an $s$-connected groupoid integrating a coupling Dirac structure has a presymplectic 2 -form which is itself a coupling form.

3A. Presymplectic groupoids. In the sequel, we will denote by $\mathcal{G} \rightrightarrows M$ a Lie groupoid, with source and target maps $s, t: \mathcal{G} \rightarrow M$, identity section $\iota: M \rightarrow \mathcal{G}$, $m \mapsto \mathbf{1}_{m}$, and inversion $i: \mathcal{G} \rightarrow \mathcal{G}, x \mapsto x^{-\mathbf{1}}$. The composition of two arrows, denoted by $x \cdot y$, is only defined provided $\boldsymbol{s}(x)=\boldsymbol{t}(y)$.

We will denote by $p_{A}: A \rightarrow M$ a Lie algebroid with Lie bracket $[\cdot, \cdot]_{A}$ and anchor $\sharp: A \rightarrow T M$. Given a Lie groupoid $\mathcal{G}$, the corresponding Lie algebroid has underlying vector bundle $A(\mathcal{G}):=\operatorname{kerd}_{\iota(M)} s$ and anchor $\sharp:=\mathrm{d}_{\iota(M)} t$. The sections of $A(\mathcal{G})$ can be identified with the right invariant vector fields on $\mathcal{G}$, and this determines the Lie bracket on sections of $A(\mathcal{G})$. A groupoid that arises in this way is called integrable.

Not every Lie algebroid $p_{A}: A \rightarrow M$ is integrable. However, there always exists a topological groupoid $\mathcal{G}(A)$ with source 1-connected fibers, that formally "integrates" $A$, called the Weinstein groupoid of $A$. Moreover, $A$ is integrable if and 
only if $\mathcal{G}(A)$ is smooth, in which case $A(\mathcal{G}(A))$ is canonically isomorphic to $A$; see [Crainic and Fernandes 2003; 2011].

Let us recall briefly the construction of $\mathcal{G}(A)$. More details can be found in [loc. cit.]. An A-path is a path $a: I \rightarrow A$ such that:

$$
\sharp a(t)=\frac{\mathrm{d}}{\mathrm{d} t} p_{A}(a(t)) .
$$

We denote by $P(A)$ the space of $A$-paths (up to reparametrization), and we set $\boldsymbol{s}(a):=p_{A} \circ a(0)$ and $\boldsymbol{t}(a):=p_{A} \circ a(1)$. On the space $P(A)$, there is an equivalence relation $\sim$, called $A$-homotopy, that preserves the multiplication. The Weinstein groupoid is the quotient of $P(A)$ by $A$-homotopies:

$$
\mathcal{G}(A):=P(A) / \sim \text {. }
$$

Given an $A$-path $a$, we denote its $A$-homotopy class by $[a]_{A}$, or simply $[a]$ when no confusion seems possible.

When a Lie algebroid arises from a geometric structure, the corresponding Lie groupoid usually inherits some extra geometric structure. In the case of Dirac structures $L$, the Weinstein groupoid $\mathcal{G}(L)$ comes equipped with a multiplicative presymplectic form; see [Bursztyn et al. 2004].

Definition 3.1. A 2-form $\Omega \in \Omega^{2}(\mathcal{G})$ is multiplicative if

$$
m^{*} \Omega=\operatorname{pr}_{1}^{*} \Omega+\operatorname{pr}_{2}^{*} \Omega,
$$

where $m: \mathcal{G}^{(2)} \rightarrow \mathcal{G}$ is the multiplication of composable arrows and $\operatorname{pr}_{i}: \mathcal{G}^{(2)} \rightarrow \mathcal{G}$ is the projections onto factor $i$. A presymplectic groupoid is a Lie groupoid endowed with a multiplicative 2 -form $\Omega$ such that

$$
\operatorname{ker} \Omega_{x} \cap \operatorname{ker}(\mathrm{d} s)_{x} \cap \operatorname{ker}(\mathrm{d} t)_{x}=\{0\}, \quad \text { for all } x \in M .
$$

Roughly speaking, Dirac structures integrate to presymplectic groupoids.

Theorem 3.2 [Bursztyn et al. 2004]. Let L be a Dirac structure on a manifold M. If $L$ is integrable, then $\mathcal{G}(L)$ has a naturally induced multiplicative presymplectic form such that the map $(\boldsymbol{t}, \boldsymbol{s}):(\mathcal{G}(L), \Omega) \rightarrow\left(M \times M, L \times L^{\mathrm{op}}\right)$ is $f$-Dirac.

The aforementioned multiplicative presymplectic form $\Omega$ on $\mathcal{G}(L)$ is related to sections of $L$ in the following way: for any $X \in T \mathcal{G}$ and any pair of sections $\eta=(v, \alpha), \xi=(w, \beta)$ in $\Gamma(L)$,

$$
\Omega(\stackrel{\leftarrow}{\eta}, X)=-\alpha\left(s_{*} X\right), \quad \Omega(\vec{\xi}, X)=\beta\left(\boldsymbol{t}_{*} X\right),
$$

where we denoted by $\grave{\eta}$ the left invariant vector field on $\mathcal{G}(L)$ associated to $\eta$ and by $\vec{\xi}$ the right invariant vector field associated to $\xi$. Also, source and target fibers 
turn out to be presymplectically orthogonal:

$$
\Omega(\overleftarrow{\eta}, \vec{\xi})=0
$$

Finally, if $\left(S, \omega_{S}\right)$ is the presymplectic leaf of $(M, L)$ through $x \in M$, then the map $\boldsymbol{t}: \boldsymbol{s}^{-1}(x) \rightarrow S$ defines a principal $G_{x}$-bundle, and

$$
i_{s^{-1}(x)}^{*} \Omega=\left.\boldsymbol{t}\right|_{s^{-1}(x)} ^{*} \omega_{S},
$$

where $i_{s^{-1}(x)}: s^{-1}(x) \hookrightarrow \mathcal{G}(L)$ denotes the inclusion.

Note that, given an integrable Dirac structure $(M, L)$, there can be other presymplectic groupoids $\left(\mathcal{G}, \Omega_{\mathcal{G}}\right)$ integrating $(M, L)$ besides $(\mathcal{G}(L), \Omega)$. However, if $\left(\mathcal{G}, \Omega_{\mathcal{G}}\right)$ has source connected fibers, then there is a covering Lie groupoid homomorphism $\Phi:(\mathcal{G}(L), \Omega) \rightarrow\left(\mathcal{G}, \Omega_{\mathcal{G}}\right)$ with $\Phi^{*} \Omega_{\mathcal{G}}=\Omega$.

3B. Couplings integrate to couplings. Assume that $L$ is an integrable coupling Dirac structure on a fibration $p: E \rightarrow B$. The anchor $\sharp: L \rightarrow T E$ is a Lie algebroid morphism that integrates to the groupoid morphism $\mathcal{G}(L) \rightarrow \Pi(E)$ which associates to the homotopy class of an $L$-path the homotopy class of its base path. On the other hand, $p_{*}: T E \rightarrow T B$ is a Lie algebroid morphism whose integration $\Pi(E) \rightarrow \Pi(B)$ is the morphism $[\gamma] \mapsto[p \circ \gamma]$. We will denote the composition of this two groupoid morphisms by $\tilde{p}: \mathcal{G}(L) \rightarrow \Pi(B)$.

Now the morphism $\tilde{p}: \mathcal{G}(L) \rightarrow \Pi(B)$ integrates the Lie algebroid morphism $p_{*} \circ \sharp: L \rightarrow T B$. Since $p_{*} \circ \sharp$ is surjective on the fibers, by the coupling condition, we see that $\tilde{p}: \mathcal{G}(L) \rightarrow \Pi(B)$ is a submersion, which is not necessarily surjective.

Proposition 3.3. Suppose that $L$ is an integrable coupling Dirac structure on a fibration $p: E \rightarrow B$ and the induced Ehresmann connection is complete. Then, $\tilde{p}: \mathcal{G}(L) \rightarrow \Pi(B)$ is surjective, so it is a fibration.

Proof. Given $[\gamma] \in \Pi(B)$, where $\gamma: I \rightarrow B$ is a smooth path, completeness allows us to lift $\gamma$ to a horizontal path $\tilde{\gamma}: I \rightarrow E$. Since $\tilde{\gamma}^{\prime}(t) \in \operatorname{Im} \sharp$, we can find an $L$-path $a: I \rightarrow L$ with base path $\tilde{\gamma}$. Then, $\tilde{p}([a])=[\gamma]$.

Remark 3.4. One can show that if a locally trivial fibration $p: E \rightarrow M$ admits a complete Ehresmann connection, then $p_{*}: \Pi(E) \rightarrow \Pi(B)$ is also locally trivial and carries an induced Ehresmann connection. It follows then that if $L$ is an integrable coupling Dirac structure on a fibration $p: E \rightarrow B$ and the induced Ehresmann connection is complete, then $\tilde{p}: \mathcal{G}(L) \rightarrow \Pi(B)$ is also a locally trivial fibration.

From now on, we will make the implicit assumption that our coupling Dirac structures have complete induced connections. This is the case, for example, if the fibers are compact. 
Theorem 3.5. Let $L$ be a coupling Dirac structure on $p: E \rightarrow B$. If $L$ is integrable, then the multiplicative presymplectic form $\Omega$ on $\mathcal{G}(L)$ is fiber nondegenerate for the fibration

$$
\tilde{p}: \mathcal{G}(L) \rightarrow \Pi(B),
$$

obtained by integrating the Lie algebroid morphism $p_{*} \circ \sharp: L \rightarrow T B$.

Proof. Let us denote by $\operatorname{Ver}_{\mathcal{G}_{L}}:=\operatorname{ker} \tilde{p}$. We only need to check that the nondegeneracy condition (4) holds:

$$
\left(\operatorname{Ver}_{\mathcal{G}(L)} \oplus \operatorname{Ver}_{\mathcal{G}(L)}^{0}\right) \cap \operatorname{Graph} \Omega=\{0\} .
$$

First notice that since $\tilde{p}$ is obtained by composing the groupoid maps

$$
\mathcal{G}(L) \rightarrow \Pi(E) \rightarrow \Pi(B),
$$

it follows that $(X, \alpha) \in T \mathcal{G}(L) \oplus T^{*} \mathcal{G}(L)$ lies in $\operatorname{Ver}_{\mathcal{G}(L)} \oplus \operatorname{Ver}_{\mathcal{G}(L)}^{0}$ if and only if it satisfies

$$
\left(\boldsymbol{s}_{*} \times \boldsymbol{t}_{*}\right)(X) \in \operatorname{Ver} \times \operatorname{Ver} \text { and } \alpha \in\left(\boldsymbol{s}^{*} \times \boldsymbol{t}^{*}\right)\left(\operatorname{Ver}^{0} \times \operatorname{Ver}^{0}\right) .
$$

Let $g \in \mathcal{G}(L)$ be the base point of $(X, \alpha)$, and set $x:=\boldsymbol{s}(g) \in E$ and $y:=t(g) \in E$. The second condition shows that $\alpha \in \boldsymbol{s}^{*}\left(\operatorname{Ver}_{x}^{0}\right)+\boldsymbol{t}^{*}\left(\operatorname{Ver}_{x}^{0}\right)$, so there exists $a_{0} \in \operatorname{Ver}_{x}^{0}$ and $a_{1} \in \operatorname{Ver}_{y}^{0}$ such that $\alpha=\boldsymbol{s}^{*} a_{0}-\boldsymbol{t}^{*} a_{1}$. It follows from the first condition that

$$
\left(\boldsymbol{s}_{*} X, a_{0}\right) \in \operatorname{Ver}_{x} \oplus \operatorname{Ver}_{x}^{0} \quad \text { and } \quad\left(\boldsymbol{t}_{*} X, a_{1}\right) \in \operatorname{Ver}_{y} \oplus \operatorname{Ver}_{y}^{0} .
$$

Thus, for any $(X, \alpha) \in\left(\operatorname{Ver}_{\mathcal{G}(L)} \oplus \operatorname{Ver}_{\mathcal{G}(L)}^{0}\right) \cap \operatorname{Graph} \Omega$, we must have $\left(\boldsymbol{s}_{*} X, a_{0}\right) \in L_{x}$ and $\left(\boldsymbol{t}_{*} X, a_{1}\right) \in L_{y}$, since $\boldsymbol{s} \times \boldsymbol{t}$ is a forward Dirac map. By the fiber nondegeneracy condition of $L$, we conclude that $\left(\boldsymbol{s}_{*} X, a_{0}\right)=0$ and $\left(\boldsymbol{t}_{*} X, a_{1}\right)=0$.

It follows that $\alpha=\boldsymbol{s}^{*} a_{0}-\boldsymbol{t}^{*} a_{1}=0$ and that $X \in \operatorname{ker} \boldsymbol{s}_{*} \cap \operatorname{ker} \boldsymbol{t}_{*}$. Since $(X, \alpha) \in$ Graph $\Omega$, we conclude that $X \in \operatorname{ker} \boldsymbol{s}_{*} \cap \operatorname{ker} \boldsymbol{t}_{*} \cap \operatorname{ker} \Omega$. The nondegeneracy condition of $\Omega$ (see Definition 3.1) shows that we must also have $X=0$.

Remark 3.6. For each $b \in B$, the fiber $\tilde{p}^{-1}\left(\mathbf{1}_{b}\right)$ is a Lie subgroupoid of $\mathcal{G}(L)$ over the fiber $E_{b}:=p^{-1}(b)$, and the restriction of $\Omega$ to the fiber is symplectic: it is a symplectic groupoid integrating the vertical Poisson structure $\left(E_{b}, \pi_{b}\right)$; the fact that ker $p_{*} \circ \sharp=\sharp^{-1}$ (Ver) is identified with Graph $\pi_{V}=$ Ver* as a Lie algebroid is a consequence of Proposition 2.10. The kernel of $\tilde{p}$ is also a Lie subgroupoid of $\mathcal{G}(L)$ over $E$ of a special kind, called a fibered symplectic groupoid, which we will study in Section 4A.

If $\left(\mathcal{G}, \Omega_{\mathcal{G}}\right)$ is another presymplectic groupoid integrating $(E, L)$ with source connected fibers, then we claim that there is also a fibration $\bar{p}: \mathcal{G} \rightarrow \mathcal{G}_{B}$, where $\mathcal{G}_{B}$ is a certain Lie groupoid integrating $T B$; in fact, since $\mathcal{G}$ has source connected fibers, there is a covering homomorphism $\Phi: \mathcal{G}(L) \rightarrow \mathcal{G}$, whose kernel $\mathcal{N} \subset \mathcal{G}(L)$ 
is an embedded bundle of normal subgroups. Its image $\tilde{p}(\mathcal{N}) \subset \pi_{1}(B)$ is also an embedded bundle of normal subgroups and so the quotient $\mathcal{G}_{B}:=\pi_{1}(B) / \tilde{p}(\mathcal{N})$ is another Lie groupoid integrating $T B$. Moreover, we obtain a groupoid morphism $\bar{p}: \mathcal{G} \rightarrow \mathcal{G}_{B}$ from $\tilde{p}: \mathcal{G}(L) \rightarrow \pi(B)$ by passing to the quotient. We then obtain as a corollary of Theorem 3.5 :

Corollary 3.7. Let $L$ be a coupling Dirac structure on $E \rightarrow B$. If $\left(\mathcal{G}, \Omega_{\mathcal{G}}\right)$ is any source connected presymplectic groupoid integrating $L$, then $\Omega_{G}$ is a coupling form relative to the fibration $\bar{p}: \mathcal{G} \rightarrow \mathcal{G}_{B}$, the unique Lie groupoid homomorphism integrating the Lie algebroid morphism $\sharp \circ p_{*}$.

3C. Integration of the geometric data. Let $L$ be an integrable coupling Dirac structure on $p: E \rightarrow B$, with associated geometric data $\left(\pi_{V}, \Gamma, \omega_{H}\right)$, and $(\mathcal{G}, \Omega)$ a source connected presymplectic groupoid integrating $L$. According to the results of the previous section, $\Omega$ is a coupling form relative to a fibration $\bar{p}: \mathcal{G} \rightarrow \mathcal{G}_{B}$, which is a Lie groupoid homomorphism integrating the Lie algebroid morphism $p_{*} \circ \sharp$. We denote by $\left(\Omega_{V}, \tilde{\Gamma}, \Omega_{H}\right)$ the corresponding geometric data.

One can obtain the geometric data of the coupling multiplicative 2-form $\Omega$ in terms of the geometric data of the coupling Dirac structure $L$ as follows:

Proposition 3.8 (integration of the geometric data). The geometric data $\left(\Omega_{V}, \tilde{\Gamma}, \Omega_{H}\right)$ for $\Omega$ is related to the geometric data $\left(\pi_{V}, \Gamma, \omega_{H}\right)$ for $L$ in the following way:

(i) $\left(\mathcal{G}_{E_{b}}, \Omega_{E_{b}}\right):=\left(\tilde{p}^{-1}\left(\mathbf{1}_{b}\right), i_{b}^{*} \Omega_{V}\right)$ is a symplectic Lie groupoid over $E_{b}$, which integrates $\left.\pi_{V}\right|_{E_{b}}$, where $i_{b}: \tilde{p}^{-1}\left(\mathbf{1}_{b}\right) \hookrightarrow \mathcal{G}$ is the inclusion.

(ii) The connection $\tilde{\Gamma}$ has horizontal lift given by

$$
\mathrm{H}(v, w)=\overleftarrow{h}^{*}(v)-\vec{h}^{*}(w),
$$

where $h^{*}$ denotes the cohorizontal lift.

(iii) Under the natural identification $T_{g} \mathcal{G}_{B}=T_{t(g)} B \times T_{s(g)} B$, the horizontal form $\Omega_{H}$ is given by

$$
\Omega_{H}\left(H\left(v_{1}, w_{1}\right), H\left(v_{2}, w_{2}\right)\right)=\omega_{H}\left(h\left(v_{1}\right), h\left(v_{2}\right)\right) \circ \boldsymbol{t}-\omega_{H}\left(h\left(w_{1}\right), h\left(w_{2}\right)\right) \circ \boldsymbol{s} .
$$

Proof. Item (i) was already discussed in Section 3B (see Remark 3.6).

To prove item (ii), consider an element $(v, w) \in T_{g} \mathcal{G}_{B}=T_{\boldsymbol{t}(g)} B \times T_{\boldsymbol{s}(g)} B$. Using the expression $h^{*}(v):=\left(h(v), i_{h(v)} \omega_{H}\right)$ for the cohorizontal lifts, one checks that the right hand term in (17) projects onto $(v, w)$ and lies in $\operatorname{Ver}_{G}^{\perp \Omega_{L}}$. By uniqueness, it must coincide with $\mathrm{H}(v, w)$. 
Finally, expression (18) for the horizontal 2-form follows by straightforward computation, using the general properties of multiplicative 2-forms:

$$
\begin{aligned}
\Omega_{H}\left(\mathrm{H}\left(v_{1}, w_{1}\right), \mathrm{H}\left(v_{2}, w_{2}\right)\right) \\
=\Omega\left(\hat{h}^{*}\left(v_{1}\right), h^{*}\left(v_{2}\right)\right)+\Omega\left(\overrightarrow{h^{*}}\left(w_{1}\right), \overrightarrow{h^{*}}\left(w_{1}\right)\right) \\
=\left\langle\left(h\left(v_{1}\right), \eta_{v_{1}}\right),\left(h\left(v_{2}\right), \eta_{v_{2}}\right)\right\rangle_{-} \circ \boldsymbol{s}-\left\langle\left(h\left(w_{1}\right), \eta_{w_{1}}\right),\left(h\left(w_{2}\right), \eta_{w_{2}}\right)\right\rangle_{-} \circ \boldsymbol{t} \\
=\omega_{H}\left(h\left(v_{1}\right), h\left(v_{2}\right)\right) \circ \boldsymbol{t}-\omega_{H}\left(w_{1}, w_{2}\right) \circ \boldsymbol{s} .
\end{aligned}
$$

Remark 3.9. the groupoid geometric data $\left(\Omega_{V}, \tilde{\Gamma}, \Omega_{H}\right)$ has a multiplicative nature:

- The fiberwise symplectic forms are multiplicative 2-form on the vertical groupoids ker $\tilde{p}$.

- The Ehresmann connection $\mathrm{H} \rightrightarrows$ Hor is a multiplicative distribution, since it is a subgroupoid of $T G \rightrightarrows T E$ over Hor $\subset T E$.

- Similarly, Equation (18) indicates that $\Omega_{H}$ is a multiplicative 2-form. There are several ways of expressing this multiplicativity. For example, one may say that for any pair of composable arrows $\left(v_{1}, w_{1}\right),\left(v_{2}, w_{2}\right) \in \operatorname{Hor}^{(2)}$, based at the same composable arrow $\left(g_{1}, g_{2}\right) \in \mathcal{G}^{(2)}$,

$$
\Omega_{H}\left(m_{\mathrm{Hor}}\left(v_{1}, w_{1}\right), m_{\mathrm{Hor}}\left(v_{2}, w_{2}\right)\right)=\Omega_{H}\left(v_{1}, v_{2}\right)+\Omega_{H}\left(w_{1}, w_{2}\right) .
$$

One may also say that the composition Hor $\rightarrow$ Hor* $\rightarrow T^{*} G$ is a groupoid morphism, where the first map is contraction by $\Omega_{H}$ and the second one is the inclusion coming from the splitting $T G=\operatorname{Ver} \oplus$ Hor.

Observing that $\Omega$ is fiber nondegenerate for both $p \circ \boldsymbol{s}$ and $p \circ \boldsymbol{t}$, we obtain:

Corollary 3.10. For each $b \in B$, the presymplectic groupoid $(\mathcal{G}, \Omega)$ and the symplectic groupoid $\left(\mathcal{G}_{E_{b}}, \Omega_{E_{b}}\right)$ are Morita equivalent presymplectic groupoids:

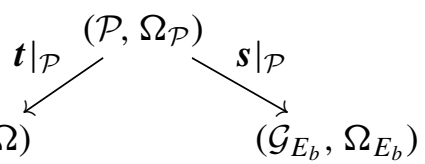

where $\mathcal{P}:=s^{-1}\left(E_{b}\right)$ and $\Omega_{\mathcal{P}}:=i_{\mathcal{P}}^{*} \Omega$, with $i_{\mathcal{P}}: \mathcal{P} \hookrightarrow \mathcal{G}$ denoting the inclusion.

\section{Integration of the Yang-Mills-Higgs phase space}

In [Brahic and Fernandes 2014], we have proposed an integration procedure for a Yang-Mills-Higgs phase space. This procedure consists in forming a certain hamiltonian quotient which is hard to make sense out of for arbitrary coupling Dirac 
structures, since it will involve an infinite dimensional reduction. In this section, we give a different approach to integrating a Yang-Mills-Higgs phase space.

This new construction of the integration of a Yang-Mills-Higgs phase space $E=P \times{ }_{G} F$ associated with a triple $(P, G, F)$ and a choice of connection $\theta: T P \rightarrow \mathfrak{g}$ involves the following steps:

(i) Integrate the Poisson structure on the fiber $\left(F, \pi_{F}\right)$ to a symplectic groupoid $\mathcal{F} \rightrightarrows F$.

(ii) Integrate the vertical Poisson structure Ver* to a fibered symplectic groupoid $\mathcal{G}_{V}=P \times_{G} \mathcal{F} \rightrightarrows E$.

(iii) Integrate the principal $G$-bundle $P \rightarrow B$ to the gauge groupoid $\mathcal{G}(P) \rightrightarrows B$.

(iv) Let the gauge groupoid $\mathcal{G}(P) \rightrightarrows B$ act on the fibered groupoid $\mathcal{G}_{V} \rightrightarrows E \rightarrow B$, yielding a semidirect product groupoid $\mathcal{G}(P) \ltimes G_{V} \rightrightarrows E$.

(v) Finally, integrate the Yang-Mills phase space, forming a quotient

$$
\mathcal{G}(L)=\mathcal{G}(P) \ltimes \mathcal{G}_{V} / \mathcal{C},
$$

where $\mathcal{C}$ is a certain curvature groupoid.

The next paragraphs describe these constructions.

4A. Fibered symplectic groupoids. We discuss the first two integration steps above. For this, we recall briefly from [Brahic and Fernandes 2008] a few notions about fibered symplectic groupoids.

4A1. Fibered groupoids. Let us fix a base $B$. We have the category Fib of fibrations over $B$, where the objects are the fibrations $p: E \rightarrow B$ and the morphisms are the fiber preserving maps over the identity.

A fibered groupoid is an internal groupoid in Fib, i.e., an internal category where every morphism is an isomorphism. This means that both the total space $\mathcal{G}_{V}$ and the base $E$ of a fibered groupoid are fibrations over $B$ and all structure maps are fibered maps. For instance, the source and the target maps are fiber preserving maps over the identity:

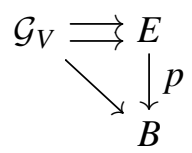

It follows that any orbit of $\mathcal{G}_{V}$ lies in a fiber of $p: E \rightarrow B$. In fact, $\left.\mathcal{G}_{V}\right|_{E_{b}}:=$ $(p \circ s)^{-1}(b)=(p \circ t)^{-1}(b)$ is a Lie groupoid over $E_{b}$.

The infinitesimal version of a fibered Lie groupoid $\mathcal{G}_{V} \rightrightarrows E \rightarrow B$ is a fibered Lie algebroid $A_{V} \rightarrow E \rightarrow B$. This means $\pi: A_{V} \rightarrow E$ is a Lie algebroid, the vector 
bundle projection is map of fibrations:

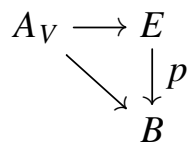

and the image of the anchor $\sharp_{V}$ takes value in the vertical bundle Ver $\subset T E$. There is an obvious Lie functor from fibered groupoids to fibered algebroids.

A elementary way to obtain a fibered groupoid/algebroid is by using a principal bundle whose structure group acts on a Lie groupoid/algebroid by automorphisms. Then we have the following:

Proposition 4.1. Given a principal $G$-bundle $P$ and an action $\boldsymbol{A}: G \rightarrow \operatorname{Aut}(\mathcal{F})$ of $G$ on a Lie groupoid $\mathcal{F} \rightrightarrows F$ by Lie groupoid automorphisms, the associated bundle $\mathcal{G}_{V}:=P \times_{G} \mathcal{F}$ carries a natural structure of a fibered Lie groupoid over $E:=P \times{ }_{G} F$. The corresponding fibered Lie algebroid is $P \times_{G} A(\mathcal{F}) \rightarrow E$.

Proof. The associated bundle $P \times_{G} \mathcal{F}$ is given by equivalence classes $[u: a]$ of couples $(u, a) \in P \times \mathcal{F}$ under the relation $[u: a]=\left[u g^{-1}, \boldsymbol{A}_{g}(a)\right]$ for all $g \in G$. The source and targets map $s, t: P \times{ }_{G} \mathcal{F} \rightarrow P \times{ }_{G} F$, given by

$$
\boldsymbol{s}[u: a]:=[u: s(a)] \text { and } \boldsymbol{t}[u: a]:=[u: t(a)],
$$

are easily checked to be well defined. Then, we define a composition by setting $\left[u^{\prime}: a^{\prime}\right] \cdot[u: a]=\left[u, \boldsymbol{A}_{g}\left(a^{\prime}\right) \cdot a\right]$, where $g$ is the unique element of $G$ such that $u^{\prime}=u g$. Once we check that it is independent of $g$, we can write

$$
\left[u: a^{\prime}\right] \cdot[u: a]=\left[u: a \cdot a^{\prime}\right],
$$

which makes it straightforward to obtain a groupoid structure $P \times_{G} \mathcal{F} \rightrightarrows P \times_{G} F$, with inverse $[u: a]^{-1}=\left[u: a^{-1}\right]$ and units $\mathbf{1}_{[u: x]}=\left[u: \mathbf{1}_{x}\right]$.

Remark 4.2. As a basic observation, note that each fiber of $P \times{ }_{G} \mathcal{F}$ comes naturally equipped with the structure of a Lie groupoid over the corresponding fiber of $P \times{ }_{G} F$, clearly isomorphic to the model $\mathcal{F} \rightrightarrows F$.

4A2. Poisson fibrations. We now apply these constructions to integrate Poisson fibrations into fibered symplectic groupoids.

Definition 4.3. A Poisson fibration $p: E \rightarrow B$ is a locally trivial fiber bundle, with fiber type a Poisson manifold $\left(F, \pi_{F}\right)$ and with structure group a subgroup $G \subset \operatorname{Diff}_{\pi}(F)$. When $\pi$ is symplectic the fibration is called a symplectic fibration.

The fibers $E_{b}:=p^{-1}(b)$ of a Poisson fibration come with an induced Poisson structure $\pi_{E_{b}}$ that glue to a Poisson structure $\pi_{V}$ on the total space of the fibration, so that $\pi_{E_{b}}=\left.\pi_{V}\right|_{E_{b}}$. 
The bivector field $\pi_{V}$ is vertical; that is, it takes values in $\bigwedge^{2}$ Ver $\subset \bigwedge^{2} T E$. Hence, the fibers $\left(E_{b}, \pi_{E_{b}}\right)$ become Poisson submanifolds of $\left(E, \pi_{V}\right)$.

It is important to distinguish $\pi_{V}$ as a vertical Poisson structure from its underlying Poisson structure on $E$. In particular, the Lie algebroid structure associated to $\pi_{V}$ as a vertical Poisson structure is defined on the covertical bundle Ver*, rather than on $T^{*} E$. The corresponding bracket and anchor are given by (13) and (12). Clearly, this is a fibered version of the usual construction, which we formalize as follows:

Definition 4.4. A fibered symplectic groupoid is a fibered Lie groupoid $\mathcal{G}_{V}$ whose fiber type is a symplectic groupoid $(\mathcal{F}, \omega)$.

Therefore, if $\mathcal{G}_{V}$ is a fibered symplectic groupoid over $B$, then $p \circ \boldsymbol{s}=p \circ \boldsymbol{t}$ : $\mathcal{G}_{V} \rightarrow B$ is a symplectic fibration, and each symplectic fiber $\left.\mathcal{G}_{V}\right|_{E_{b}}$ is a symplectic groupoid over the corresponding fiber $E_{b}$.

Proposition 4.5. The base $E \rightarrow B$ of a fibered symplectic groupoid $\mathcal{G}_{V} \rightrightarrows E \rightarrow B$ has a natural structure of a Poisson fibration.

Conversely, a Poisson fibration whose fiber type is an integrable Poisson manifold, integrates to a fibered symplectic groupoid. In fact, standard facts about integration of Lie algebroids yield the following (see [Brahic and Fernandes 2008] for details):

Theorem 4.6. Let $p: E \rightarrow B$ be a Poisson fibration with fiber type $\left(F, \pi_{F}\right)$ an integrable Poisson manifold. There exists a unique (up to isomorphism) source 1-connected fibered symplectic groupoid integrating Ver*.

Remark 4.7. The integration of $\pi_{V}$ as a Poisson fibration and as a Poisson structure differ since $\mathcal{G}\left(\right.$ Ver $\left.^{*}\right)$ has only dimension $2 \operatorname{dim}(F)+\operatorname{dim}(B)$.

4B. Action groupoids. Next we will discuss steps (iii) and (iv) in the integration of Yang-Mills phase spaces. We describe an action of the gauge groupoid of a principal bundle on an associated fibered groupoid, and the resulting action groupoid.

4B1. Action of a Lie groupoid on a fibered Lie groupoid. Given a fibered groupoid $\mathcal{G}_{V} \rightrightarrows E \stackrel{p}{\rightarrow} B$, the gauge groupoid is the transitive (infinite dimensional) groupoid

$$
\operatorname{Gau}\left(\mathcal{G}_{V}\right):=\left\{\left.\left.\mathcal{G}_{V}\right|_{E_{b}} \stackrel{g}{\rightarrow} \mathcal{G}_{V}\right|_{E_{b^{\prime}}}: g \text { is a Lie groupoid isomorphism }\right\},
$$

with source $s(g)=b$, target $t(g)=b^{\prime}$, and with the obvious composition.

Definition 4.8. An action of a groupoid $\mathcal{G} \rightrightarrows B$ on a fibered groupoid $\mathcal{G}_{V} \rightrightarrows E \rightarrow B$ is a Lie groupoid homomorphism $\Phi: \mathcal{G} \rightarrow \operatorname{Gau}\left(\mathcal{G}_{V}\right)$.

There is an associated semidirect action groupoid $\mathcal{G} \ltimes \mathcal{G}_{V} \rightrightarrows E$ associated to such an action, whose space of arrows is defined as

$$
\mathcal{G} \ltimes \mathcal{G}_{V}:=\mathcal{G}_{s} \times_{p \circ t} \mathcal{G}_{V}=\left\{(g, a) \in \mathcal{G} \times \mathcal{G}_{V}:\left.a \in \mathcal{G}_{V}\right|_{E_{s}}(g)\right\} .
$$


The source and target are given by $\boldsymbol{s}(g, a):=\boldsymbol{s}(a)$ and $\boldsymbol{t}(g, a):=\boldsymbol{t}\left(\Phi_{g}(a)\right)$, the units by $\mathbf{1}_{x}=\left(\mathbf{1}_{p(x)}, \mathbf{1}_{x}\right)$, the inverses by $(g, a)^{-1}=\left(g^{-1}, \Phi_{g}(a)^{-1}\right)$, and the composition by

$$
\left(g_{2}, a_{2}\right) \cdot\left(g_{1}, a_{1}\right)=\left(g_{2} \cdot g_{1}, \Phi_{g_{1}}^{-1}\left(a_{2}\right) \cdot a_{1}\right) .
$$

When both $\mathcal{G}$ and $\mathcal{G}_{V}$ are Lie groupoid, we say that the action is smooth whenever $\mathcal{G} \ltimes \mathcal{G}_{V}$ is a Lie groupoid for the obvious manifold structure.

In order to define the infinitesimal counterpart of this action groupoid, for a fibered Lie algebroid $A_{V} \rightarrow E$, define

$$
\operatorname{Der}_{B}\left(A_{V}\right):=\left\{D \in \operatorname{Der}\left(A_{V}\right): \text { the symbol of } D \text { is } p_{*} \text {-projectable }\right\}
$$

There is a well defined map $\rho: \operatorname{Der}_{B}\left(A_{V}\right) \rightarrow \mathfrak{X}(B), D \mapsto p_{*} X_{D}$, where $X_{D}$ denotes the symbol of $D$. Note that $\operatorname{Der}_{B}\left(A_{V}\right)$ is a $C^{\infty}(B)$-module by the formula $(f . D)(\alpha):=f D(\alpha)$. In fact, $\operatorname{Der}_{B}\left(A_{V}\right)$ is a Lie algebra over $C^{\infty}(B)$ and $\rho$ a $C^{\infty}(B)$-linear morphism of Lie algebras. In this work, we will always assume that $A_{V}$ is locally trivial, so that $\rho$ is surjective.

Definition 4.9. An action of a Lie algebroid $A \rightarrow B$ on a fibered Lie algebroid $A_{V} \rightarrow E \rightarrow B$ is a $C^{\infty}(B)$-linear Lie algebra morphism $\mathcal{D}: \Gamma(A) \rightarrow \operatorname{Der}_{B}\left(A_{V}\right)$ covering the anchor map, that is, such that $\sharp_{A}=\rho \circ \mathcal{D}$.

Given such an action, $A \ltimes A_{V}:=p^{*} A \oplus A_{V}$ comes naturally with the structure of a Lie algebroid over $E$. The bracket and anchor are given by

$$
\begin{gathered}
\sharp(v, \alpha):=X_{\mathcal{D}_{v}}+\sharp_{V}(\alpha), \\
{[v, w]:=[v, w]_{A}, \quad[\alpha, \beta]:=[\alpha, \beta]_{A_{V}}, \quad[v, \alpha]:=\mathcal{D}_{v}(\alpha) .}
\end{gathered}
$$

for any sections $\alpha, \beta \in \Gamma\left(A_{V}\right)$ and $v, w \in \Gamma(A)$ seen as sections of $A \ltimes A_{V}$. The above brackets and anchor naturally extend to arbitrary sections of $A \ltimes A_{V}$ since $\gamma(A)$ generates $\Gamma\left(p^{*} A\right)$ as a $C^{\infty}(E)$-module.

Definition 4.10. Given an action $\mathcal{D}: \Gamma(A) \rightarrow \operatorname{Der}_{B}\left(A_{V}\right)$ of $A$ on $A_{V}$, we call the Lie algebroid $A \ltimes A_{V}$ described above the action Lie algebroid.

Remark 4.11. If $A_{V}$ is the Lie algebroid of a fibered Lie groupoid $\mathcal{G}_{V}$, then $\operatorname{Der}_{B}\left(A_{V}\right)$ can be thought of as the Lie algebroid of Gau $\left(\mathcal{G}_{V}\right)$. Indeed, any smooth action $\Phi: \mathcal{G} \rightarrow \operatorname{Gau}\left(\mathcal{G}_{V}\right)$ differentiates to an action $\mathcal{D}: A \rightarrow \operatorname{Der}_{B}\left(A_{V}\right)$. Moreover, $A \ltimes A_{V} \rightarrow E$ is the Lie algebroid of $\mathcal{G} \ltimes \mathcal{G}_{V} \rightrightarrows E$.

In order to integrate an infinitesimal action $\mathcal{D}: \Gamma(A) \rightarrow \operatorname{Der}_{B}\left(A_{V}\right)$, note however that we need to assume that $A$ acts by complete lifts, meaning that the symbol of $\mathcal{D}_{\alpha}$ is a complete vector field on $E$ for any $\alpha \in \Gamma(A)$. 
4B2. Actions of principal bundles on fibered Lie groupoids. Given a principal $G$-bundle $P$, recall that its gauge groupoid $\mathcal{G}(P) \rightrightarrows B$ has spaces of arrows the associated bundle $P \times_{G} P$.

Denoting by $\left[u_{2}: u_{1}\right]$ the equivalence class of a couple $\left(u_{2}, u_{1}\right) \in P \times P$, the source and target of $\mathcal{G}(P)$ are defined by $s\left(\left[u_{2}: u_{1}\right]\right):=q\left(u_{1}\right), \boldsymbol{t}\left(\left[u_{2}: u_{1}\right]\right):=q\left(u_{2}\right)$, and the composition is well defined by setting:

$$
[w: v] \cdot[v: u]=[w: u] .
$$

The inverses are given by $[v: u]^{-1}=[v: u]$ and identities by $\mathbf{1}_{x}=[u: u]$.

The groupoid $\mathcal{G}(P) \rightrightarrows B$ is transitive, and its isotropy groups fit into a Lie group bundle Iso $P \rightarrow B$ that canonically identifies with the associated bundle $P \times_{G} G$ by the injection $[u: h] \mapsto[u h: u]$. Note that the same goes for neutral components, namely Iso ${ }_{P}^{\circ}=P \times{ }_{G} G^{\circ}$.

We will be interested in the $s$-simply connected groupoid $\widetilde{\mathcal{G}}(P)$ corresponding to $\mathcal{G}(P)$ rather than $\mathcal{G}(P)$ itself. The principal bundle corresponding to $\widetilde{\mathcal{G}}(P)$ has total space the universal cover $\widetilde{P}$ of $P$. When $G$ is connected, the structure group $\bar{G}$ of $\widetilde{P}$ fits into an exact sequence:

$$
1 \rightarrow \operatorname{Im} \partial_{2} \rightarrow \widetilde{G} \rightarrow \bar{G} \rightarrow 1,
$$

where $\partial_{2}: \pi_{2}(B) \rightarrow \pi_{1}(G)$ is the boundary operator in the homotopy sequence of the projection $P \rightarrow B$. This means one can always assume that $\pi_{1}(G)=\operatorname{Im} \partial_{2}$, provided one chooses to work with $\widetilde{\mathcal{G}}(P)$ instead of $\mathcal{G}(P)$.

Finally, the Lie algebroid associated to $\mathcal{G}(P)$ is usually denoted by $T P / G$. It is a vector bundle over $B$ whose sections are the $G$-invariant vector fields on $P$, and who fits in the Atiyah sequence:

$$
\operatorname{ker} \sharp \hookrightarrow T P / G \rightarrow T B .
$$

Proposition 4.12. Let $P$ be a principal $G$-bundle and $A: G \rightarrow \operatorname{Aut}(\mathcal{F})$ an action of $G$ on a Lie groupoid $\mathcal{F} \rightrightarrows F$ by Lie groupoid automorphisms. Then, there is a natural action of the gauge groupoid $\mathcal{G}(P)$ on the associated fibered Lie groupoid $\mathcal{G}_{V}:=P \times_{G} \mathcal{F} \rightrightarrows E:=P \times_{G} F$.

Proof. Define $\Phi: \mathcal{G}(P) \rightarrow \operatorname{Gau}\left(\mathcal{G}_{V}\right)$ by $\Phi_{\left[u_{2}: u_{1}\right]}([u: a]):=\left[u_{2} g: a\right]$, where $g$ is the unique element of $G$ such that $u_{1} g=u$. After checking that $\Phi$ is well defined, notice that a more convenient formula for $\Phi$ is simply:

$$
\Phi_{[v: u]}([u: a])=[v: a] \quad \text { for } u, v \in P, a \in \mathcal{F} .
$$

This makes it straightforward to check that $\Phi$ indeed takes values in $\operatorname{Gau}\left(\mathcal{G}_{V}\right)$, and that it is a groupoid morphism. 
Proposition 4.13. With the same assumptions as in Proposition 4.12, the action groupoid $\mathcal{G} \ltimes \mathcal{G}_{V} \rightrightarrows E$ identifies with the quotient $(P \times P \times \mathcal{F}) / G$, where $G$ acts on $P \times P \times F$ diagonally: $[v: u: a]=\left[v g: u g: \boldsymbol{A}_{g}^{-1}(a)\right]$, for any $g \in G$. Moreover, under this identification, the structure maps are given as follows:

- the source and target map are

$$
\boldsymbol{s}[v: u: a]=[u: s(a)] \text { and } \boldsymbol{t}[v: u: a]=[v: t(a)],
$$

- the unit at a point $[u: x]$, where $x \in F, u \in P$, is

$$
\mathbf{1}_{[u: x]}=\left[u: u: \mathbf{1}_{x}\right],
$$

- the inverses are

$$
[v: u: a]^{-1}=\left[u: v: a^{-1}\right],
$$

- and the composition is

$$
\left[w: v: a^{\prime}\right] \cdot[v: u: a]=\left[w: u: a^{\prime} \cdot a\right] .
$$

Proof. By the construction of the Section 4B1, an arrow in $\mathcal{G}(P) \ltimes \mathcal{G}_{V}$ is a couple ([ $\left.\left.u_{2}: u_{1}\right],[u: a]\right)$, where $q(u)=q\left(u_{1}\right)$. Since there exists a unique $g \in G$ such that $u_{1} g=u$, we can always assume that $u_{1}=u$ and the identification easily follows. The formulas for the structure maps then come from Proposition 4.12 and the construction of the semidirect product.

4B3. Action groupoid of a Poisson fibration. Let $E=P \times_{G} F \rightarrow B$ be a Poisson fibration associated with a principal $G$-bundle $p: P \rightarrow B$ and an action of $G$ on an integrable Poisson manifold $\left(F, \pi_{F}\right)$ by Poisson diffeomorphisms. The results above show that one obtains an action groupoid as follows.

First, we consider the source connected symplectic groupoid $\mathcal{F} \rightrightarrows F$ integrating $\left(F, \pi_{F}\right)$. The $G$-action on $F$ by Poisson diffeomorphisms lifts to Lie groupoid action $\boldsymbol{A}: G \rightarrow \operatorname{Aut}(\mathcal{F})$ by groupoid automorphisms; see, e.g., [Fernandes et al. 2009]. Therefore, according to Propositions 4.12 and 4.13, there is a natural action of the gauge groupoid $\mathcal{G}(P) \rightrightarrows B$ on the associated fibered Lie groupoid $\mathcal{G}_{V}:=P \times_{G} \mathcal{F} \rightrightarrows E \rightarrow B$, giving rise to an action Lie groupoid $\mathcal{G}(P) \ltimes \mathcal{G}_{V} \rightrightarrows E$.

According to the preceding discussion (see Remark 4.11), the Lie algebroid of the action Lie groupoid $\mathcal{G}(P) \ltimes \mathcal{G}_{V} \rightrightarrows E$ has underlying vector bundle

$$
p^{*} A \ltimes A_{V}=p^{*}(T P / G) \ltimes \operatorname{Ver}^{*} .
$$

To determine the bracket and the anchor, we need to find the Lie algebra homomorphism $\mathcal{D}: \Gamma(T P / G) \rightarrow \operatorname{Der}_{B}\left(\operatorname{Ver}^{*}\right)$. Since Ver* identifies naturally with the associated bundle Ver* $=P \times_{G} T^{*} F$ and since the action of $G$ on $T^{*} F$ is naturally lifted from the $G$-action on $F$, it follows that $\mathcal{D}$ associates to each $G$-invariant vector field $X$ in $P$ the Lie derivative of the vector field $X_{E} \in \mathfrak{X}(E)$, induced by the natural 
action on $T^{*} P / G$ on $E$. In other words, $\mathcal{D}_{v}$ coincides with the Lie derivative of its own symbol:

$$
\mathcal{D}_{X}(\alpha)=\mathcal{L}_{X_{E}} \alpha \quad \text { for } X \in \Gamma(T P / G), \alpha \in \Gamma\left(\text { Ver }^{*}\right),
$$

where $X_{E}$ is the projection on $E=P \times{ }_{G} F$ of the vector field $(X, 0) \in \mathfrak{X}(P \times F)$. It follows that if $X, Y$ denote $G$-invariant vector fields in $P$ and $\alpha, \beta \in \Gamma$ (Ver*), then the anchor of $p^{*}(T P / G) \ltimes$ Ver* is given by

$$
\sharp(X, \alpha):=X_{E}+\pi_{V}^{\sharp}(\alpha),
$$

while the bracket takes the form

$$
[X, Y]_{A \ltimes A_{V}}:=[X, Y], \quad[\alpha, \beta]_{A \ltimes A_{V}}:=[\alpha, \beta], \quad[X, \alpha]_{A \ltimes A_{V}}:=\mathcal{L}_{X_{E}}(\alpha) .
$$

4C. Integrability of Yang-Mills-Higgs phase spaces. We consider now the last steps in the construction of the integration of Yang-Mills-Higgs phase space. So now we assume that we have

- $p: P \rightarrow B$ a principal $G$-bundle;

- $\left(F, \pi_{F}\right)$ a Poisson manifold;

- $G \times F \rightarrow F$ a hamiltonian $G$-action on $\left(F, \pi_{F}\right)$ with equivariant moment map $J_{F}: F \rightarrow \mathfrak{g}^{*}$.

Each choice of a principal connection $\theta: T P \rightarrow \mathfrak{g}$ yields a coupling Dirac structure on $E=P \times{ }_{G} F$.

The fact that the action is hamiltonian implies that the $G$ action on the algebroid $T^{*} F$ is prehamiltonian, with premoment map (see ):

$$
\begin{aligned}
\psi: \mathfrak{g} \ltimes F & \longrightarrow T^{*} F \\
(\xi, m) & \longmapsto \mathrm{d}_{m}\langle J, \xi\rangle .
\end{aligned}
$$

Therefore, by Theorem A.19, $\psi$ integrates to a groupoid morphism

$$
\Psi: G^{\circ} \ltimes F \rightarrow \mathcal{F},
$$

where

$$
\mathcal{F}:=\Sigma(F) / \tilde{\Psi}\left(\pi_{1}(G) \ltimes F\right) .
$$

We will assume that $\tilde{\Psi}\left(\pi_{1}(G) \times F\right)$ is embedded in $\Sigma(F)$, so that $\mathcal{F}$ is smooth. Clearly, $\mathcal{G}_{V}:=P \times{ }_{G} \mathcal{F}$ is a symplectic groupoid integrating Ver* $=P \times{ }_{G} T^{*} F$.

The $G$-action on $F$ lifts to a Lie groupoid action $A: G \rightarrow \operatorname{Aut}(\mathcal{F})$, so we can apply the construction of the previous subsection: we obtain an action groupoid $\mathcal{G}(P) \ltimes \mathcal{G}_{V} \rightrightarrows E$. 
Definition 4.14. The curvature subgroupoid, denoted by $\mathcal{C} \rightrightarrows E$, is the subgroupoid $\mathcal{C} \subset \mathcal{G}(P) \ltimes \mathcal{G}_{V}$ given by:

$$
\mathcal{C}:=\operatorname{Graph}\left(\Psi_{P} \circ i\right) \subset \mathcal{G}(P) \ltimes \mathcal{G}_{V},
$$

where $\Psi_{P}:$ Iso $_{P}^{\circ} \times_{B} E \rightarrow \mathcal{G}_{V}$ is obtained by fibrating $\Psi: G^{\circ} \ltimes F \rightarrow \mathcal{F}$ along $P$ and $i: \mathrm{Iso}^{\circ} \rightarrow$ Iso $^{\circ}$ denotes the inversion.

More explicitly, with the notations of Proposition 4.13, the curvature groupoid $\mathcal{C}$ is given by

$$
\mathcal{C}:=\left\{\left[u h^{-1}: u: \Psi(h, x)\right] \in \mathcal{G}(P) \ltimes \mathcal{G}_{V}: u \in P, h \in G^{\circ}, x \in F\right\} .
$$

Proposition 4.15. The curvature groupoid $\mathcal{C} \rightrightarrows E$ is a wide, normal, completely intransitive subgroupoid of $\mathcal{G}(P) \ltimes \mathcal{G}_{V}$.

Proof. The result follows using the expression (22) for $\mathcal{C}$, the compositions rules in Proposition 4.13 and Equation (32) in Theorem A.19. The fact that $\mathcal{C}$ is a subgroupoid is rather straightforward. In order to see that it is normal, we pick any $\left[u h^{-1}: u: \Psi(h, x)\right] \in \mathcal{C}$ and $[v: u: a] \in \mathcal{G}(P) \ltimes \mathcal{G}_{V}$ which are composable, i.e., such that $x=s(a)$, and we find that

$$
[v: u: a] \cdot\left[u h^{-1}: u: \Psi(h, x)\right] \cdot[v: u: a]^{-1}=\left[v h^{-1}: v: \Psi(h, x)\right],
$$

is an element in $\mathcal{C}$.

Finally, putting all together, we conclude the following:

Theorem 4.16. Suppose that $(P, G, F)$ is a classical Yang-Mills-Higgs setting and $\theta: T P \rightarrow \mathfrak{g}$ is a principal connection. Let $L$ be the corresponding coupling Dirac structure on $E=P \times_{G} F$ and assume that

(i) the Poisson manifold $\left(F, \pi_{F}\right)$ is integrable, and

(ii) the groupoid $\tilde{\Psi}\left(\pi_{1}(G) \times F\right)$ is embedded in $\Sigma(F)$.

Then, the quotient groupoid $\mathcal{G}(P) \ltimes \mathcal{G}_{V} / \mathcal{C}$ integrates $(E, L)$.

Proof. As we saw above, the Lie algebroid of $\mathcal{G}(P) \ltimes \mathcal{G}_{V}$ is given by $A \ltimes A_{V}$, where $A=T P / G$ and $A_{V}=$ Ver*. Furthermore, the principal connection induces a splitting of the Atiyah sequence, and we have an identification $T P / G \simeq T B \oplus \operatorname{ker} \sharp_{A}$. With this identification, the Lie algebroid $A_{\mathcal{C}}$ of $\mathcal{C}$ lies in

$$
T P / G \ltimes \operatorname{Ver}^{*} \simeq\left(T B \oplus \operatorname{ker} \sharp_{A}\right) \ltimes \operatorname{Ver}^{*}
$$

as

$$
A_{\mathcal{C}}=\left\{(0, \xi,-\psi(\xi)) \in\left(T B \oplus \operatorname{ker} \sharp_{A}\right) \ltimes \operatorname{Ver}^{*}: \xi \in \operatorname{ker} \sharp_{A}\right\},
$$

and the quotient ( $\left.T B \oplus \operatorname{ker} \sharp_{A}\right) \ltimes \operatorname{Ver}^{*} / A_{\mathcal{C}}$ identifies with $T B \times{ }_{B}$ Ver*, with canonical projection given by $\pi(X, \xi, \alpha)=(X, \alpha+\psi(\xi))$. It now follows from expressions 
(20) and (21) for the anchor and the brackets that the Lie algebroid structure on $A \ltimes$ Ver* descends to a Lie algebroid structure on $T B \times{ }_{B} A_{V}$ whose brackets and anchor are the same as those given in Proposition 2.10. Hence $A \ltimes \operatorname{Ver}^{*} / A_{\mathcal{C}}$ and $L$ are isomorphic as Lie algebroids.

For the smoothness of the quotient, we observe that $\mathcal{G}(P) \ltimes \mathcal{G}_{V} / \mathcal{C}$ can also be thought of as an "associated bundle" $\mathcal{G}(P) \ltimes_{\text {Iso }_{P}^{\circ}} \mathcal{G}_{V}$. Indeed, there is an action of the bundle of Lie groups Iso ${ }_{P}^{\circ}$ on $\mathcal{G}(P) \ltimes \mathcal{G}_{V}$ which can be described as follows. On the one hand, the Lie groupoid morphism $\Psi$ induces an action $\lambda$ of $G^{\circ}$ on $\mathcal{F}$ by left multiplication: $\lambda_{h}(a):=\Psi(h, x) \cdot a$, where $h \in G^{\circ}$ and $x:=s(a)$. Fibering along $P$, we obtain an action of the bundle of Lie groups Iso $_{P}^{\circ}$ on $\mathcal{G}_{V}$ :

$$
\lambda_{[u: h]}^{P}([u: a]):=[u: \Psi(h, x) \cdot a] .
$$

Here we use the identification Iso $_{P}^{\circ} \simeq P \times_{G} G^{\circ}$ to write an element of Iso $_{P}^{\circ}$ as a pair $[u: h]$. Note that this action is well defined by (33). On the other hand, Iso $_{P}^{\circ}$ acts on $\mathcal{G}(P)$ by right multiplication, which is a proper and free action. The two actions together give a proper and free action of $\operatorname{Iso}_{P}^{\circ}$ on $\mathcal{G}(P) \ltimes \mathcal{G}_{V}$ :

$$
g \cdot(b, a):=\left(b g^{-1}: \lambda_{g}^{P}(a)\right) .
$$

and the quotient is the "associated bundle" $\mathcal{G}(P) \ltimes_{\text {Iso }_{P}^{\circ}} \mathcal{G}_{V}$.

We claim that $\mathcal{G}(P) \ltimes_{\text {Iso }_{P}^{\circ}} \mathcal{G}_{V}$ can be identified with $\mathcal{G}(P) \ltimes \mathcal{G}_{V} / \mathcal{C}$. This follows by observing that any $g \in \mathrm{Iso}_{P}^{\circ}$ can be written as $g=[u: u h] \in \mathcal{G}(P)$ so that (see Theorem A.19):

$$
g \cdot(b, a)=\left(b g^{-1}, \lambda_{g}^{P}(a)\right)=(b, a) \cdot c
$$

where $c:=\left(\left[u h^{-1}: u: \Psi(h)\right]\right) \in \mathcal{C}$. Since the assignment $c \leftrightarrow g$ is one-to-one, the two quotients coincide.

Remark 4.17. Consider the Hopf fibration $P=\mathbb{S}^{3} \rightarrow \mathbb{S}^{2}$, seen as an $\mathbb{S}^{1}$-principal bundle, and $F=\mathbb{R}$ acted upon trivially with momentum map $f: F \rightarrow \mathbb{R}$ any smooth function, as in Example 2.7. Then, the second condition in Theorem 4.16 fails if $f$ has a critical point as explained in Example A.20.

Theorem 4.16 shows that the groupoid structure of $\mathcal{G}(L)$ does not depend on the choice of the principal $G$-bundle connection. In other words, two coupling Dirac structures associated with Yang-Mills data with the same principal $G$-bundle and hamiltonian $G$-action, but different principle bundle connections, give rise to the same Lie groupoid. Note, however, that the presymplectic forms will be distinct, as it is clear from their geometric data given in Proposition 3.8.

We can also give an explicit description of the presymplectic form $\Omega$ on $G(L)$, as follows. First, we use Proposition 4.13 to identify $\mathcal{G}(P) \ltimes \mathcal{G}_{V} \simeq(P \times P \times \mathcal{F}) / G$. We then construct a presymplectic form $\widetilde{\Omega}$ on $\mathcal{G}(P) \ltimes \mathcal{G}_{V}$ : we have a closed 2-form 
on $P \times P \times \mathcal{F}$ given by

$$
\widetilde{\Omega}:=p_{\mathcal{F}}^{*} \Omega_{\mathcal{F}}+\mathrm{d}\langle\theta, \bar{\mu}\rangle_{1}-\mathrm{d}\langle\theta, \bar{\mu}\rangle_{2},
$$

where $p_{\mathcal{F}}: P \times P \times \mathcal{F} \rightarrow \mathcal{F}$ is the projection, and $\mathrm{d}\langle\theta, \bar{\mu}\rangle_{i}$ denotes the closed 2-form in $\Omega^{2}(P \times P \times \mathcal{F})$ obtained by differentiating the 1-form $\alpha_{i} \in \Omega^{1}(P \times P \times \mathcal{F})$ given by

$$
\left.\alpha_{i}\right|_{\left(u_{1}, u_{2}, g\right)}\left(v_{1}, v_{2}, w\right):=\left\langle\left.\theta\right|_{u_{i}}\left(v_{i}\right), \bar{\mu}(g)\right\rangle .
$$

Here, $\bar{\mu}: \mathcal{F} \rightarrow \mathfrak{g}^{*}$ denotes the moment map for the lifted $G$-action on $\mathcal{F}$, so that $\bar{\mu}=\mu \circ \boldsymbol{t}-\mu \circ \boldsymbol{s}$. One checks easily that the closed 2 -form $\widetilde{\Omega}$ is basic for the $G$-action on $P \times P \times \mathcal{F}$, so it descends to a multiplicative 2 -form in the quotient $(P \times P \times \mathcal{F}) / G \simeq \mathcal{G}(P) \ltimes \mathcal{G}_{V}$.

Finally, one checks that resulting multiplicative 2-form on $\mathcal{G}(P) \ltimes \mathcal{G}_{V}$ further descends to the quotient $\mathcal{G}(P) \ltimes \mathcal{G}_{V} / \mathcal{C}$, giving a closed, multiplicative 2-form $\Omega_{\mathcal{G}}$ satisfying the nondegeneracy condition (15). A more-or-less tedious computation shows that the target map $t:\left(\mathcal{G}(L), \Omega_{\mathcal{G}}\right) \rightarrow(E, L)$ is a forward Dirac map. Summarizing this discussion, we have:

Corollary 4.18. Under the conditions of Theorem 4.16, the presymplectic form on the groupoid $G(L)=\mathcal{G}(P) \ltimes \mathcal{G}_{V} / \mathcal{C}$ is the quotient of the closed 2-form

$$
\widetilde{\Omega}:=p_{\mathcal{F}}^{*} \Omega_{\mathcal{F}}+\mathrm{d}\langle\theta, \bar{\mu}\rangle_{1}-\mathrm{d}\langle\theta, \bar{\mu}\rangle_{2} .
$$

The integrability conditions in Theorem 4.16 can be made more explicit. On the one hand, the integrability of the fiber type $\left(F, \pi_{F}\right)$ follows from general theory developed in [Crainic and Fernandes 2004] and can be expressed in terms of monodromy maps $\partial: \pi_{2}(S, x) \rightarrow \mathcal{G}\left(\mathfrak{g}_{x}\right)$, where $S$ is the symplectic leaf of $F$ through $x$ and $\mathfrak{g}_{x}=\left.\operatorname{ker} \pi_{F}^{\sharp}\right|_{x}$ is the isotropy Lie algebra at $x$. On the other hand, condition (ii) can be treated by the same methods as in [Brahic and Fernandes 2014, Section 4.3], and one gets another monodromy type map $\pi_{1}(G) \rightarrow \mathcal{F}_{m}$ controlling (ii). This will be treated elsewhere.

\section{Integration of coupling Dirac structures II}

A general coupling Dirac structure may not come from a principal bundle with structure group a finite dimensional Lie group. For instance, this is the case if the holonomy group induced by the connection (i.e., the group spanned by the holonomy along loops in the base) is not a finite dimensional subgroup of the Poisson automorphisms of the fiber. In such cases, one needs a formulation of the construction of Section $4 \mathrm{C}$ which avoids infinite dimensional reductions. In this section, we will take advantage of the fact that $L$ fits into a Lie algebroid extension, to reformulate the construction given in Section $4 \mathrm{C}$, without any mentioning to 
these infinite dimensional group quotients. We follow the ideas of [Brahic 2010] in order to describe $L$-paths and $L$-homotopies.

Recalling that Ver* $=\operatorname{Graph}\left(\pi_{V}\right)$, we know that $L$ is a Lie algebroid extension

$$
\text { Ver* } \hookrightarrow L \rightarrow T B,
$$

which splits. The notion of holonomy makes sense for any Lie algebroid extension with a splitting (see [op. cit., Section 2.1]), and in our situation, given a $T B$-path $\dot{\gamma}_{B} \in P(T B)$, the holonomy is a Lie algebroid morphism

$$
\Phi_{\gamma_{B}}:\left.\left.\operatorname{Ver}^{*}\right|_{E_{\gamma_{B}(0)}} \rightarrow \operatorname{Ver}^{*}\right|_{E_{\gamma_{B}(1)}} .
$$

It will be useful to restrict $\gamma_{B}$ to a path $[0, t] \rightarrow T B$, where $t \in[0,1]$. The corresponding holonomy will then be

$$
\Phi_{t, 0}^{\gamma_{B}}:\left.\left.\operatorname{Ver}^{*}\right|_{E_{\gamma_{B}(0)}} \rightarrow \operatorname{Ver}^{*}\right|_{E_{\gamma_{B}(t)}} .
$$

In the case of a coupling Dirac structure, there is another notion of holonomy to be taken into account, namely, the one induced by the usual Ehresmann connection Hor. Given a path $\gamma_{B}:[0,1] \rightarrow B$ it gives rise to a holonomy map

$$
\phi^{\gamma_{B}}: E_{\gamma_{B}(0)} \rightarrow E_{\gamma_{B}(1)} .
$$

Again, restricting $\gamma_{B}$ to a path $[0, t] \rightarrow B$ the corresponding holonomy will be denoted

$$
\phi_{t, 0}^{\gamma_{B}}: E_{\gamma_{B}(0)} \rightarrow E_{\gamma_{B}(t)} .
$$

The two holonomies are related in a simple way:

Proposition 5.1. The holonomy $\Phi_{t, 0}^{\gamma_{B}}$ induced by the connection Graph $\left(\omega_{H}\right)$ on $L$ is related to the holonomy $\phi_{t, 0}^{\gamma_{B}}$ induced by Hor on TE by

$$
\Phi_{t, 0}^{\gamma_{B}}=\left(\phi_{0, t}^{\gamma_{B}}\right)^{*} .
$$

Proof. The result follows directly from the identification Ver* $=\operatorname{Graph}\left(\pi_{V}\right)$ and from the particular form of the bracket given in Proposition 2.10.

Recall that a Lie algebroid extension is called a fibration whenever the Ehresmann connection is complete [Brahic and Zhu 2011]. It follows from Proposition 5.1 that (10) is a fibration whenever the Ehresmann connection Hor is complete. In the sequel, we will always assume that this is the case.

5A. Splitting L-paths and L-homotopies. We see from Proposition 2.9 that any $L$-path $a$ over $\gamma:=p_{L} \circ a$ decomposes uniquely as a sum:

$$
a(t)=h^{*}\left(\dot{\gamma}_{B}(t)\right)_{\gamma(t)}+a_{V}(t)
$$


where $\gamma_{B}:=p \circ \gamma$. In this decomposition, neither $t \mapsto h^{*}\left(\dot{\gamma}_{B}(t)\right)_{\gamma(t)}$, nor $t \mapsto a_{V}(t)$ is an $L$-path in general. However, it is possible to "split" the paths in $P(L)$ into horizontal and vertical parts, as follows:

Proposition 5.2 (splitting $L$-paths). Let $L$ be a coupling Dirac structure on a fibration $p: E \rightarrow B$. If the associated connection $\Gamma$ is complete, then there is an isomorphism of Banach manifolds:

$$
\begin{aligned}
& P(L) \longrightarrow P(T B){ }_{s} \times{ }_{t \circ p} P\left(\text { Ver }^{*}\right), \\
& a \longmapsto \quad\left(\dot{\gamma}_{B}, \tilde{a}\right) \text {, }
\end{aligned}
$$

where the couple $\left(\dot{\gamma}_{B}, \tilde{a}\right)$ is defined by

$$
\dot{\gamma}_{B}:=\mathrm{d} p \circ \sharp a, \quad \tilde{a}_{t}:=a_{V}(t) \circ \mathrm{d} \phi_{t, 0}^{\gamma_{B}},
$$

where $\phi_{t, 0}^{\gamma_{B}}: E_{\gamma_{B}(0)} \rightarrow E_{\gamma_{B}(t)}$ denotes the holonomy along $\gamma_{B}$.

Proof. This follows from [Brahic 2010, Proposition 4.1] and Proposition 5.1.

One should think of the couple $\left(\dot{\gamma}_{B}, \tilde{a}\right)$ as a concatenation of $L$-paths of the form $h^{*}\left(\dot{\gamma}_{B}\right) \cdot \tilde{a}$. Here, $h^{*}\left(\dot{\gamma}_{B}\right)$ denotes the $L$-path defined by

$$
h^{*}\left(\dot{\gamma}_{B}\right)(t):=h^{*}\left(\dot{\gamma}_{B}(t)\right)_{\phi_{t, 1}^{\gamma_{B}}(y)},
$$

where $y=s(a)$. Notice that the $L$-path (25) is different from the horizontal component appearing in (23) since the base paths are different. In particular, $h^{*}\left(\dot{\gamma}_{B}\right)$ as defined in (25) is always an $L$-path by construction.

Then Proposition 5.2 can be illustrated in a simple way as follows:

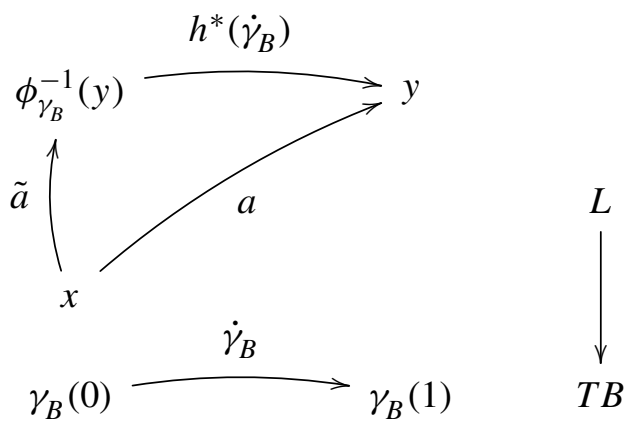

In fact, it can be proved that $a$ is $L$-homotopic to the concatenation $h^{*}\left(\dot{\gamma}_{B}\right) \cdot \tilde{a}$. However, for the sake of simplicity, in this work we shall simply think of the map $a \mapsto\left(\tilde{a}, \dot{\gamma}_{B}\right)$ as an mere identification.

Recall that for any $A$-path $a$, its inverse path is the $A$-path $a^{-1}$ defined by $a^{-1}(t):=-a(1-t)$. Using Proposition 5.2, one can express the concatenation and inverses of $L$-paths as follows: 
Proposition 5.3. Under the isomorphism of Proposition 5.2, given two composable L-paths $a \simeq\left(\dot{\gamma}_{B}, \tilde{a}\right)$ and $b \simeq\left(\dot{\delta}_{B}, \tilde{b}\right)$, their concatenation is

$$
\left(\dot{\delta}_{B}, \tilde{b}\right) \cdot\left(\dot{\gamma}_{B}, \tilde{a}\right):=\left(\dot{\delta}_{B} \cdot \dot{\gamma}_{B}, \Phi_{\gamma_{B}}^{-1}(\tilde{b}) \cdot \tilde{a}\right) .
$$

Moreover, the inverse path $a^{-1}$ of a is

$$
\left(\dot{\gamma}_{B}, \tilde{a}\right)^{-1}:=\left(\dot{\gamma}_{B}^{-1}, \Phi_{\gamma_{B}}(\tilde{a})^{-1}\right) .
$$

Proof. The result follows directly from (24) and from the fact that the holonomy commutes with taking concatenation and inverse of $A$-paths.

Notice the analogy between the formula for concatenation in the previous proposition and formula (19) for the product in the action groupoid. In fact, if one thinks of $P(T B)$ as a groupoid over $B$, then the holonomy gives an action of $P(T B)$ on $P\left(\right.$ Ver $\left.^{*}\right)$ similar to the action of $\mathcal{G}(P)$ on $\mathcal{G}_{V}$ discussed in Section 4B1. For this reason, one may think of the fibered product $P(T B)_{s} \times{ }_{t \circ p} P\left(\right.$ Ver $\left.^{*}\right)$ as a semidirect product $P(T B) \ltimes P\left(\right.$ Ver $\left.^{*}\right)$.

In general, the presence of curvature prevents the fundamental groupoid $\Pi(B)$ from acting on $P\left(\right.$ Ver $\left.^{*}\right)$. However, holonomy along a path $\gamma_{B} \in P(B)$ is a Lie algebroid morphism $\Phi_{\gamma_{B}}$ : Ver* $\left.\left.\right|_{E_{\gamma_{B}(0)}} \rightarrow \operatorname{Ver}^{*}\right|_{E_{\gamma_{B}(1)}}$. Hence, it integrates to a groupoid morphism $\Phi_{\gamma_{B}}:\left.\left.\mathcal{G}\left(\right.$ Ver* $\left.^{*}\right)\right|_{E_{\gamma_{B}(0)}} \rightarrow \mathcal{G}\left(\right.$ Ver* $\left.^{*}\right)\right|_{E_{\gamma_{B}(1)}}$ that we still denote by $\Phi_{\gamma_{B}}$. Here, $\mathcal{G}\left(\right.$ Ver $\left.^{*}\right)$ denotes the Weinstein groupoid of Ver*. Finally, notice that the formulas in Proposition 5.3 still make sense when replacing Ver*-paths by their homotopy classes; therefore, we will denote by $P(T B) \ltimes \mathcal{G}$ (Ver*) the fibered product $P(T B)_{s} \times{ }_{t \circ p} \mathcal{G}\left(\right.$ Ver $\left.^{*}\right)$.

Theorem 5.4. Suppose that $L$ is a coupling Dirac structure on $E \rightarrow B$. The source 1-connected groupoid $\mathcal{G}(L)$ integrating $L$ naturally identifies with equivalence classes in $P(T B) \ltimes_{B} \mathcal{G}\left(\right.$ Ver* $\left.^{*}\right)$ under the following relation:

- $\left(\gamma_{0}, g_{0}\right) \sim\left(\gamma_{1}, g_{1}\right)$ if and only if there exists a homotopy $\gamma_{B}: I \times I \rightarrow B$, $(t, \epsilon) \mapsto \gamma_{B}^{\epsilon}(t)$ between $\gamma_{0}$ and $\gamma_{1}$, such that $g_{1}=\partial\left(\gamma_{B}, t\left(g_{0}\right)\right) \cdot g_{0}$.

Here, $\partial\left(\gamma_{B}, x_{0}\right)$ is the element in $\mathcal{G}\left(\mathrm{Ver}^{*}\right)$ represented by the Ver*-path

$$
\epsilon \longmapsto\left(\mathrm{d}_{V}\right)_{\tilde{\gamma} \epsilon}\left(\int_{0}^{1}\left(\phi_{s, 0}^{\gamma_{B}^{\epsilon}}\right)^{*} \omega_{H}\left(\gamma_{B}\right)_{s, \epsilon} \mathrm{d} s\right) \in \operatorname{Ver}_{\tilde{\gamma}(\epsilon)}^{*},
$$

where $\tilde{\gamma}(\epsilon):=\Phi_{\gamma_{B}^{\epsilon}}^{-1} \circ \Phi_{\gamma_{B}^{0}}\left(x_{0}\right)$ and

$$
\omega_{H}\left(\gamma_{B}\right)_{s, \epsilon}:=\omega_{H}\left(h\left(\frac{\mathrm{d} \gamma_{B}}{\mathrm{~d} t}(s, \epsilon)\right), h\left(\frac{\mathrm{d} \gamma_{B}}{\mathrm{~d} \epsilon}(s, \epsilon)\right)\right) \in C^{\infty}\left(E_{\gamma_{B}(s, \epsilon)}\right)
$$


One may illustrate the homotopy condition appearing in Theorem 5.4 in the following way:
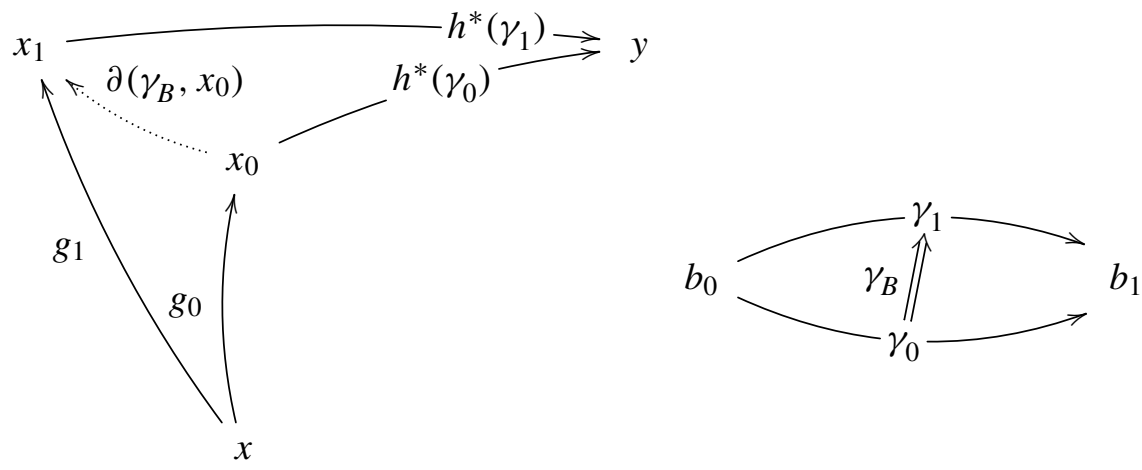

Example 5.5. Let us consider the case where $\pi_{V}$ is the trivial Poisson structure. This occurs, for instance, if $L$ is the restriction of a regular Dirac structure to a tubular neighborhood $E \rightarrow B$ of one of its leaves $B$. Then $\mathcal{G}$ (Ver*) is a bundle of Lie groups that identifies with Ver* with its additive structure. Furthermore, it follows from the curvature identity (9) that the connection is flat, so we have a genuine action of the fundamental groupoid $\Pi(B)$ on Ver* Up to a cover of $B$, we may assume that $E$ is trivial as a representation of $\Pi(B)$. This means that $E$ can be identified with $B \times F$ in such a way that the holonomy along any path is the identity:

$$
\phi_{s, 0}^{\gamma}=\operatorname{id}_{F}:\{\gamma(0)\} \times F \longrightarrow\{\gamma(1)\} \times F .
$$

It follows that the horizontal and vertical distributions are respectively given by Hor $=T B \times F$ and Ver $=B \times T F$ in the decomposition $T E=T B \oplus T F$. Hence, $\omega_{H}$ can be seen as a family of 2-forms on $B$ parametrized by $F$, and the leaves of $L$ are of the form $B \times\{x\}$ with presymplectic form $\left.\omega_{H}\right|_{B \times\{x\}}$, where $x \in F$.

The homotopy condition appearing in Theorem 5.4 can then be expressed as follows: two elements $\left(\gamma_{0}, g_{0}\right)$ and $\left(\gamma_{1}, g_{1}\right)$ in $P(T B) \times T_{x_{0}}^{*} F$ are homotopic if and only if there exists a $T B$-homotopy $\gamma_{B}: I^{2} \mapsto B$ between $\gamma_{0}$ and $\gamma_{1}$ such that

$$
g_{1}-g_{0}=\left(\mathrm{d}_{V}\right)_{x_{0}} \int_{\gamma_{B}} \omega_{H},
$$

where we integrate $\omega_{H}$ along $\gamma_{B}$ as a 2-form with values in $C^{\infty}(F)$. In order to obtain the above formula, we simply replace $\phi_{0, s}^{\gamma_{B}^{\epsilon}}$ by $\mathrm{id}_{F}$ in (26) and then we use the fact that, $T^{*} F$ being a bundle of abelian groups, any path in $T_{x_{0}}^{*} F$ can be represented by a constant paths. This amounts in (26) to average with respect to the $\epsilon$ variable. In particular, when $F=\mathbb{R}$, we recover the leafwise prequantization Lie algebroids and the homotopy condition appearing in [Crainic 2004]. 
Notice also that, applying the resulting 1-form to a vector $X_{x_{0}} \in T F$, one gets a geometrical interpretation of $g_{1}-g_{0}$ as the variation of the presymplectic area of $\gamma_{B}$ in the vertical directions:

$$
\left\langle\mathrm{d}_{V} \int_{\gamma_{B}} \omega_{H}, X_{x_{0}}\right\rangle=\left.\frac{\mathrm{d}}{\mathrm{d} t}\right|_{t=0} \int_{\gamma_{B} \times\left\{\phi_{t}^{X}\left(x_{0}\right)\right\}} \omega_{H},
$$

where $\phi_{t}^{X}$ is the flow of any vector field $X \in \mathfrak{X}(F)$ extending $X_{x_{0}}$.

Remark 5.6. The construction given in Theorem 5.4 can be interpreted as an infinite dimensional analogue of the construction given in Section 4 of the groupoid integrating a Yang-Mills phase space.

For this interpretation, one considers the Poisson frame bundle (see [Brahic and Fernandes 2008]), so that we can view our coupling as an infinite dimensional Yang-Mills phase space. One needs first to reduce the structure group from the group of Poisson diffeomorphisms between a fixed fiber $E_{b_{0}}$ and any other fiber to the subgroup generated by the holonomy transformations $\Phi_{\gamma_{B}}$ along any path $\gamma_{B} \in P(B)$, with $\gamma_{B}(0)=b_{0}$. If $P \rightarrow B$ denotes the resulting principal bundle, then one can "identify" the corresponding gauge groupoid $\operatorname{Gau}(P)=P \times{ }_{B} P$ with the "groupoid" $P(T B)$. Moreover, the equivalence relation $\sim$ of Theorem 5.4 can be viewed as the equivalence relation associated with the corresponding curvature groupoid.

5B. The monodromy groupoid. We now use the constructions of [Brahic 2010; Crainic and Fernandes 2004] in order to obtain the obstructions to integrability of a coupling Dirac structure $L$.

Consider the short exact sequence of Lie algebroids

$$
\operatorname{Ver}^{*} \hookrightarrow L \rightarrow T B
$$

We obtain by integration the sequence of groupoid morphisms

$$
\mathcal{G}\left(\text { Ver }^{*}\right) \stackrel{j}{\longrightarrow} \mathcal{G}(L) \stackrel{q}{\longrightarrow} \Pi(B),
$$

where $\Pi(B)$ denotes the fundamental groupoid of $B$. Recall that $j$ and $q$ are defined at the level of paths:

$$
j\left([\tilde{a}]_{V}\right):=[i \circ a]_{L} \text { and } q\left([a]_{L}\right):=\left[p_{*} \circ \sharp(a)\right]_{T B} .
$$

for any Ver*-path $\tilde{a}: I \rightarrow$ Ver* $^{*}$ and any $L$-path $a: I \rightarrow L$. Although the sequence (27) is exact, the sequence (28) might not be exact anymore, pointing out a lack of exactness of the integration functor. However, one can always ensure the right exactness: 
Proposition 5.7. Let $L$ be a coupling Dirac structure whose induced Ehresmann connection is complete. Then, the sequence (28) is surjective at $\Pi(B)$ and exact at $\mathcal{G}(L)$.

Proof. One may see that $q$ is surjective, provided the connection is complete, by observing that, given $\left[\dot{\gamma}_{B}\right]_{T B} \in \Pi(B)$, the element $\left[h^{*}\left(\dot{\gamma}_{B}\right)\right] \in \mathcal{G}(L)$, defined by $(25)$ maps to $\left[\dot{\gamma}_{B}\right]$.

For the exactness at $\mathcal{G}(L)$ one observes that, by the definition, the elements of ker $q$ are represented by $L$-paths whose projection on $T B$ is a contractile loop. Therefore, the inclusion $\operatorname{Im} j \subset \operatorname{ker} q$ is obvious. Conversely, given an element $[a]_{L} \in \operatorname{ker} q$, represented by some $L$-path $a$, we see that $a \sim\left(\tilde{a}, \dot{\gamma}_{B}\right)$, under the identifications of Proposition 5.2, where $\gamma_{B}$ is a contractible loop based at some $b \in B$. Consider a contraction $\gamma_{B}^{\epsilon}: I^{2} \rightarrow B$ between $\gamma_{B}$ and the trivial path $0_{b}$. Then, by Theorem 5.4, we see that $\left(\tilde{a}, \dot{\gamma}_{B}\right)$ is $L$-homotopic to $\left(\partial\left(\gamma_{B}\right) \cdot \tilde{a}, 0_{b}\right)$. Since $\left(\partial\left(\gamma_{B}\right) \cdot \tilde{a}, 0_{b}\right)$ represents a Ver*-path, we conclude that $[a]_{L} \in \operatorname{Im} j$, as claimed.

It follows that (28) can only fail to be exact because of the lack of injectivity of $j$. In order to measure this failure, we introduce the following:

Definition 5.8. The monodromy groupoid associated with the fibration is the kernel of $j: \mathcal{G}\left(\right.$ Ver* $\left.^{*}\right) \rightarrow \mathcal{G}(L)$, denoted by $\mathcal{M}$.

Obviously, by construction, we have an exact sequence of groupoids

$$
\mathcal{M} \hookrightarrow \mathcal{G}\left(\text { Ver }^{*}\right) \rightarrow \operatorname{ker} q,
$$

and we can replace (28) by the exact sequence of groupoids:

$$
\mathcal{G}\left(\operatorname{Ver}^{*}\right) / \mathcal{M} \hookrightarrow \mathcal{G}\left(\text { Ver }^{*}\right) \rightarrow \Pi(B) .
$$

The kernel of this sequence $\operatorname{ker} q=\mathcal{G}\left(\operatorname{Ver}^{*}\right) / \mathcal{M}$ is a bundle of groupoids with typical fiber the neutral component of the restricted groupoid $\left.\mathcal{G}(L)\right|_{E_{b_{0}}}$ to a fiber $E_{b_{0}}$. In particular, we see that if $\mathcal{G}(L)$ is integrable, then the monodromy groupoid $\mathcal{M}$ must be embedded in $\mathcal{G}\left(\right.$ Ver $\left.^{*}\right)$.

It remains to relate $\mathcal{M}$ to the global data associated with $L$ on $E$.

Theorem 5.9. Consider a coupling Dirac structure $L$ on a fibration $E \rightarrow B$, and assume that the induced Eheresmann connection is complete. Then there exists a homomorphism

$$
\partial: \pi_{2}(B) \times_{B} E \rightarrow \mathcal{G}\left(\operatorname{Ver}^{*}\right),
$$

that makes the following sequence exact:

$$
\cdots \rightarrow \pi_{2}(B) \times{ }_{B} E \rightarrow \mathcal{G}\left(\text { Ver }^{*}\right) \rightarrow \mathcal{G}(L) \rightarrow \Pi(B) .
$$


In other words, Theorem 5.9 states that the monodromy groupoids of the fibration coincide with the image of the transgression map $\mathcal{M}=\left.\operatorname{Im} \partial\right|_{\pi_{2}(B)}$.

Proof. The map $\partial$ in Theorem 5.4, when restricted to a sphere in $B$ based at some $b \in B$ (seen as a map $\gamma_{B}: I^{2} \rightarrow B$ such that $\left.\gamma_{B}\left(\partial I^{2}\right)=\{b\}\right)$ is independent of its homotopy class; see [Brahic 2010]. Then, it follows from that reference and [Brahic and Zhu 2011] that the restriction of the map $\partial$ to $\pi_{2}(B)$ corresponds precisely to the transgression map.

Note the analogy between the monodromy groupoid described above and the monodromy groups that measure the integrability of an algebroid [Crainic and Fernandes 2003]. In fact, when $E$ is a tubular neighborhood of a leaf $B \subset E$ in a Dirac structure, the restriction $\left.\mathcal{M}\right|_{B}$ coincides, by construction, with the usual monodromy groups along $B$.

Finally, we can relate the monodromy groupoid of a coupling Dirac structure with the problem of integrability.

Theorem 5.10. Let $L$ be a coupling Dirac structure on $E \rightarrow B$ and assume that the associated connection $\Gamma$ is complete. Then, $L$ is an integrable Lie algebroid if and only if the following conditions hold:

(i) the typical Poisson fiber $\left(E_{x},\left.\pi_{V}\right|_{E_{x}}\right)$ is integrable;

(ii) the injection $\mathcal{M} \hookrightarrow \mathcal{G}\left(\right.$ Ver $\left.^{*}\right)$ is an embedding.

Proof. First, it is easily seen that since the associated Poisson fibration is locally trivial, Ver* is integrable if and only if the typical Poisson fiber $\left(E_{x},\left.\pi_{V}\right|_{E_{x}}\right)$ is integrable.

Assume now that $L$ is integrable. Then, the projection $q: \mathcal{G}(E) \rightarrow \Pi(B)$ is a smooth surjective submersion. Therefore, $\operatorname{ker} q$ is a Lie groupoid integrating Ver; in particular, the typical Poisson fiber is integrable. Furthermore, since $\operatorname{ker} q=\mathcal{G}\left(\right.$ Ver $\left.^{*}\right) / \mathcal{M}$ is smooth, $\mathcal{M}$ is necessarily embedded in $\mathcal{G}\left(\right.$ Ver $\left.^{*}\right)$.

Conversely, suppose that $\mathcal{M}$ is embedded in $\mathcal{G}\left(\right.$ Ver $\left.^{*}\right)$ and consider a sequence $\left(\xi_{n}\right) \subset \mathcal{N}(L)$ of monodromy elements of $L$ converging to a trivial path $0_{x}$. Since ker $\sharp \subset$ Ver*, one can consider the sequence $\left[\xi_{n}\right]_{V} \in \mathcal{G}\left(\right.$ Ver $\left.^{*}\right)$, where $\xi_{n}$ is considered as a constant path. By the definition [Crainic and Fernandes 2004] of the monodromy groups $\mathcal{N}(L)$ controlling the integrability of $L,\left[\xi_{n}\right]_{L} \in \mathcal{G}(L)$ is a sequence of units $\left[\xi_{n}\right]_{L}=\mathbf{1}_{x_{n}}$, therefore $\left[\xi_{n}\right]_{V} \in \mathcal{M}$. In other words, there exists a neighborhood $U$ of the identity section in $\mathcal{G}(L)$ such that $\mathcal{N}(L) \cap U \subset \mathcal{M} \cap U$. Since $\mathcal{M}$ is embedded in $\mathcal{G}$ (Ver*), it follows that there exists a neighborhood $V \subset U$ of the identity section in $\mathcal{G}(L)$ such that $\mathcal{N}(L) \cap V$ coincides with the identity section. This shows that the obstructions to integrability of $L$ vanish.

Example 5.11 (hamiltonian symplectic fibrations). Assume $L$ that is the graph of a presymplectic form. Then $L$ identifies with $T E$ as a Lie algebroid (using the 
anchor map). In particular $L$ is integrable and $\mathcal{G}(L)$ identifies with the fundamental groupoid of $E$. Let us see how one can recover this using the previous construction.

In that case, $\pi_{V}$ is the inverse of a symplectic (vertical) form. Thus, Ver* identifies with Ver as a Lie algebroid, and $\mathcal{G}\left(\right.$ Ver $\left.^{*}\right) \simeq \mathcal{G}($ Ver $)$, which is just a fibered version of the fundamental groupoid. Therefore, the transgression map becomes $\partial: \pi_{2}(B) \ltimes E \rightarrow \mathcal{G}$ (Ver) and, as easily checked, corresponds to the usual transgression map in the homotopy long exact sequence associated to the fibration $E \rightarrow B$. It follows that $\mathcal{M}_{x}$ lies in the fundamental group $\pi_{1}\left(E_{p(x)}\right)$ of the fiber through $x \in E$, and $\mathcal{M}$ is locally trivial over $E$. Now, Theorem 5.10 shows that $L$ is integrable.

In fact, if the fibers are compact, one can even show that the transgression map vanishes. Indeed, given a sphere in $B$, it follows from (26) that the loops representing the image of the transgression map are the so-called hamiltonian loops; see [McDuff and Salamon 1998]. For compact symplectic manifolds, it is a well known fact that such hamiltonian loops are always contractile.

Example 5.12 (split Poisson structures). When a coupling Dirac structure $L$ is the graph of a Poisson structure $\pi$, the decomposition (8) corresponds to a splitting $\pi=\pi_{V}+\pi_{H}$, where $\pi_{H}$ is a bivector field; see [Vorobjev 2001].

One may check that the corresponding connection has vanishing curvature if and only if $\pi_{H}$ is Poisson. The characteristic foliation of $\pi_{H}$ is then given by the integrable distribution Hor. Moreover, it follows from the curvature identity (9) that the connection is flat if and only if $\omega_{H}$ takes values in the space of Casimirs of $\pi_{V}$.

Let us assume that $\pi_{H}$ is indeed Poisson and, for the sake of simplicity, assume that $E=B \times F$ is a trivial fibration. Then, one can still interpret the elements of $\mathcal{M}$ in terms of variations of the symplectic area of spheres. First, notice that $\tilde{\gamma}^{\epsilon}$ is necessarily a trivial path since the connection is trivial. Furthermore, the integral in (26) involves

$$
\omega_{H}\left(\frac{\mathrm{d} \gamma_{B}}{\mathrm{~d} t}, \frac{\mathrm{d} \gamma_{B}}{\mathrm{~d} \epsilon}\right)
$$

which are Casimirs of the vertical Poisson structure on $F$. The resulting element in $\operatorname{Ver}_{x_{0}}^{*}$ lies in the center of the isotropy algebra at $x_{0}$. Thus, taking the corresponding Ver*-homotopy class amounts to integrating along the $\epsilon$ variable.

Example 5.13. As a particular case of Example 5.12, consider the trivial Poisson fibration $E=\mathbb{S}^{2} \times \mathfrak{s o}_{3}^{*} \rightarrow \mathbb{S}^{2}$, where $p$ is the projection onto the first factor. Let $\pi_{V}$ be the linear Poisson structure on the fibers $\mathfrak{s o}_{3}^{*}$ of the projection, and let $\operatorname{Hor}_{(b, x)}=T_{b} S^{2} \times\{x\}$ be the trivial connection. Then, $\omega_{H}$ must necessarily be of the form $\omega_{H}=f \cdot \omega$, where $\omega$ denotes the standard symplectic form on $S^{2}$ and $f$ is a Casimir of $\mathfrak{s o}_{3}^{*}$, i.e., a smooth function of the radius $r \in C^{\infty}\left(\mathfrak{s o}_{3}^{*}\right)$. 
One knows (see, e.g., [Crainic and Fernandes 2003]) that the (usual) monodromy groups of the vertical Poisson structure at some $(b, x) \in B \times \mathfrak{s o}_{3}^{*}$ are

$$
\mathcal{N}\left(\text { Ver }^{*}\right)_{(b, x)}= \begin{cases}4 \pi \mathbb{Z} \cdot \mathrm{d} r & \text { if } x \neq 0, \\ \{0\} & \text { if } x=0,\end{cases}
$$

Applying the integrability criteria of Theorem 5.10, we see that $L$ is integrable if and only if $4 \pi f^{\prime}(r)$ is a rational multiple of $4 \pi$, for any $r$. This means that $f^{\prime}$ must be constant, so $f(r)=\alpha r+\beta$, with $\alpha \in \mathbb{Q}$ and $\beta \in \mathbb{R}$.

One can also recover this result using the prequantization Lie algebroids of [Crainic 2004] associated with a product of presymplectic spheres. On each leaf, the restricted Lie algebroid $\left.L\right|_{S^{2} \times S^{2} \times\{v\}}$ is the prequantization of a product of presymplectic spheres $\left(S^{2} \times S^{2}, f^{\prime}(v) \omega \times \omega\right)$. It is well known that leaf wise, $f^{\prime}(v)$ must be a rational multiple of $\int_{S^{2}} \omega=4 \pi$.

This example shows how rigid the integrability conditions can be: in this example, the value and the derivative of $f$ at a point entirely determines the structure.

\section{Appendix}

A1. Actions on Lie groupoids and Lie algebroids. We will have to look at various actions of Lie groups and algebras on Lie groupoids and Lie algebroids. The following diagram summarizes the various possibilities:

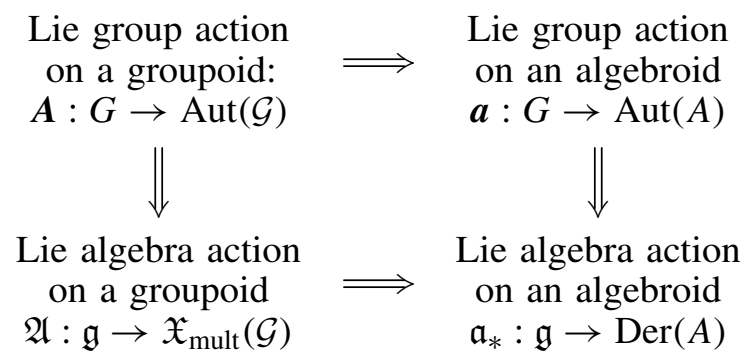

where the four corners have the following precise meaning:

- Action of a Lie group $G$ on a Lie groupoid $\mathcal{G}$ : This means a smooth action $\boldsymbol{A}: G \times \mathcal{G} \rightarrow \mathcal{G}$ such that for each $g \in G$ the map $\boldsymbol{A}_{g}: \mathcal{G} \rightarrow \mathcal{G}, x \mapsto g x$, is a Lie groupoid automorphism.

- Action of a Lie group $G$ on a Lie algebroid A: This means a smooth action $\boldsymbol{a}: G \times A \rightarrow A$ such that for each $g \in G$ the map $\boldsymbol{a}_{g}: A \rightarrow A, a \mapsto a x$, is a Lie algebroid automorphism.

- Action of a Lie algebra $\mathfrak{g}$ on a Lie groupoid $\mathcal{G}$ : This means a Lie algebra homomorphism $\mathfrak{A}: \mathfrak{g} \rightarrow \mathfrak{X}_{\text {mult }}(\mathcal{G})$, where $\mathfrak{X}_{\text {mult }}(\mathcal{G}) \subset \mathfrak{X}(\mathcal{G})$ denotes the multiplicative vector fields in $\mathcal{G}$. 
- Action of a Lie algebra $\mathfrak{g}$ on a Lie algebroid A: This means a Lie algebra homomorphism $\mathfrak{a}_{*}: \mathfrak{g} \rightarrow \operatorname{Der}(A)$, where $\operatorname{Der}(A)$ is the space of derivations of the Lie algebroid $A$.

Clearly, Lie group actions on Lie groupoids and algebroids cover ordinary Lie group actions on the base manifold. Similarly, Lie algebra actions on Lie groupoids and algebroids cover ordinary Lie algebra actions on the base manifold.

The arrows in the diagram above represent natural differentiation operations, either along the group action or along the groupoid. The explicit description is left to the reader, and then the commutativity of the diagram becomes obvious.

Under appropriate assumptions one can also invert the arrows in the diagram above, namely:

- One can invert the horizontal arrows (integrate actions on Lie algebroids to actions on Lie groupoids) if $\mathcal{G}=\mathcal{G}(A)$, the source 1-connected Lie groupoid integrating $A$.

- One can invert the vertical arrows (integrate Lie algebra actions to Lie group actions) if $G=G(\mathfrak{g})$, the source 1-connected Lie group integrating $\mathfrak{g}$, and if the infinitesimal actions are complete (the flows are defined for all $t \in \mathbb{R}$ ).

The reader should be able to fill in the details.

A2. Inner actions. Recall that a bisection $b: M \rightarrow \mathcal{G}$ is a smooth section of the source map such that $t \circ b$ is a diffeomorphism of $M$. The space $\operatorname{Bis}(\mathcal{G})$ of bisections has natural structure of a group, induced from the groupoid structure, and the map $\operatorname{Bis}(\mathcal{G}) \rightarrow \operatorname{Diff}(M), b \mapsto t \circ b$ is a morphism of groups.

The notion of inner action for Lie groupoids follows immediately from the following definitions:

- An inner Lie groupoid automorphism is a Lie groupoid automorphism $\Phi: \mathcal{G} \rightarrow \mathcal{G}$ of the form

$$
\Phi(x)=b(t(x)) \cdot x \cdot b(s(x))^{-1} .
$$

for some bisection $b: M \rightarrow \mathcal{G}$. They clearly form a subgroup $\operatorname{InnAut}(\mathcal{G}) \subset \operatorname{Aut}(\mathcal{G})$. - A inner Lie algebroid automorphism is a Lie algebroid automorphism $\phi: A \rightarrow A$ of the form

$$
\phi=\varphi_{1,0}^{D_{\alpha}},
$$

for some time dependent section $\alpha_{t} \in \Gamma(A)$. Here, $t \mapsto \varphi_{t, 0}^{D_{\alpha}}$ denotes the flow of the time dependent derivation $\left.D_{\alpha_{t}}:=\left[\alpha_{t}, \cdot\right]\right)$. They generate a subgroup $\operatorname{Inn} \operatorname{Aut}(A) \subset$ $\operatorname{Aut}(A)$.

- A multiplicative exact vector field is a multiplicative vector field $X \in \mathfrak{X}_{\text {mult }}(\mathcal{G})$ of the form

$$
X=\vec{\alpha}-\stackrel{\leftarrow}{\alpha}
$$


where $\alpha$ is a section of $A=A(\mathcal{G})$, and $\vec{\alpha}$ and $\overleftarrow{\alpha}$ are the right and left invariant vector fields in $\mathcal{G}$ determined by $\alpha$. They form a Lie subalgebra $\mathfrak{X}_{\text {exact }}(\mathcal{G}) \subset \mathfrak{X}_{\text {mult }}(\mathcal{G})$.

- An inner derivation is a Lie algebroid derivation $D \in \operatorname{Der}(A)$ of the form

$$
D=[\alpha, \cdot]_{A},
$$

for some section $\alpha \in \Gamma(A)$. They clearly form a Lie subalgebra $\operatorname{InnDer}(A) \subset \operatorname{Der}(A)$.

Now, one can define inner actions in a more-or-less obvious fashion. We obtain a diagram as above:

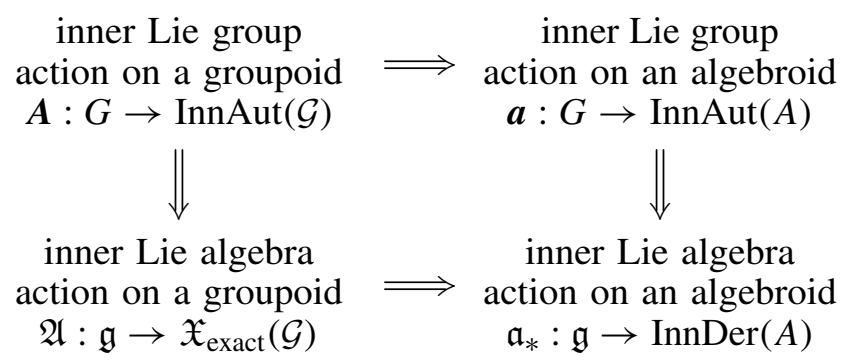

In this work, we will mainly consider inner actions associated with a Lie groupoid morphism $\Psi: G \times M \rightarrow \mathcal{G}$ given by

$$
\boldsymbol{A}_{g}(x)=\Psi(g, t(x)) \cdot x \cdot \Psi(g, s(x))^{-1}, \quad \text { for } g \in G, x \in \mathcal{G} .
$$

Notice that the map $\Psi$ covers the ordinary action $G \times M \rightarrow M$ on the base. Furthermore, one may check that $\Psi: G \times M \rightarrow \mathcal{G}$ is a Lie groupoid morphism if and only if the map $G \rightarrow \operatorname{Bis}(\mathcal{G}), g \mapsto b^{g}(x):=\Psi(g, x)$ is a group morphism covering the usual Lie group action of $G$ on $M$.

Similarly, the inner Lie algebra actions on a Lie algebroid $\mathfrak{a}_{*}: \mathfrak{g} \rightarrow \operatorname{InnDer}(A)$ will come associated with a Lie algebroid morphism $\psi: \mathfrak{g} \times M \rightarrow A$ (covering the identity on $M$ ) such that

$$
\left(\mathfrak{a}_{\xi}\right)_{*}=\left[\psi_{*}(\xi), \cdot\right]_{A}, \quad \text { for } \xi \in \mathfrak{g},
$$

where $\psi_{*}(\xi) \in \Gamma(A)$ is defined by $\psi_{*}(\xi)_{m}=\psi(\xi, m)$, for any $m \in M$. The map $\psi_{*}: \mathfrak{g} \rightarrow \Gamma(A)$ covers the ordinary Lie algebra action $\mathfrak{g} \rightarrow \mathfrak{X}(M)$ on the base. Moreover, $\psi_{*}$ is a Lie algebra morphism covering the infinitesimal action $\mathfrak{g} \rightarrow \mathfrak{X}(M)$ if and only if $\psi$ is a Lie algebroid.

Proposition A.14. Let $G \times M \rightarrow M$ be an action of a Lie group on a manifold. Then, any homomorphism $\Psi: G \ltimes M \rightarrow \mathcal{G}$ from the action Lie groupoid to a Lie groupoid $\mathcal{G}$ determines by formula (29) an inner action of $G$ on $\mathcal{G}$ that covers the action on $M$. 
Similarly, let $\mathfrak{g} \rightarrow \mathfrak{X}(M)$ be an action of a Lie algebra on a manifold. Then any homomorphism $\psi_{*}: \mathfrak{g} \ltimes M \rightarrow A$ from the action Lie algebroid to a Lie algebroid $A$ determines by formula (30) an inner action of $\mathfrak{g}$ on A that covers the infinitesimal action on $M$.

Remark A.15. Note that $\Psi$ and $\psi$ as above do not need to be morphisms in order for (29) and (30) to induce inner actions. In this paper though, we will always assume that it is the case.

The relevant notion for this work is the following:

Definition A.16. A prehamiltonian action of a Lie group $G$ on a Lie algebroid $A$ with prehamiltonian moment map $\psi_{*}: \mathfrak{g} \rightarrow \Gamma(A)$ is an action $\boldsymbol{a}: G \rightarrow \operatorname{Aut}(A)$ such that:

- $\left.\frac{\mathrm{d}}{\mathrm{d} t}\left(\boldsymbol{a}_{\exp (-t \xi)}\right)_{*}(\beta)\right|_{t=0}=\left[\psi_{*}(\xi), \beta\right]_{A}, \quad$ for $\xi \in \mathfrak{g}, \beta \in \Gamma(A)$,

- $\psi_{*}$ is a $G$-equivariant morphism of Lie algebras.

Note that the $G$-equivariance is always satisfied when $G$ is connected.

A3. Integration of inner actions. Let us now see in which circumstances one is able to invert arrows in the last diagram.

Proposition A.17. Suppose that $\mathfrak{g} \rightarrow \mathfrak{X}(M)$ is a complete Lie algebra action and $\psi: \mathfrak{g} \ltimes M \rightarrow A$ is a Lie algebroid morphism from the action Lie algebroid to a Lie algebroid $A$. For any Lie groupoid $\mathcal{G}$ integrating $A$, the associated inner action $\mathfrak{a}_{*}: \mathfrak{g} \rightarrow \operatorname{InnDer}(A)$ integrates to an inner action $A: G(\mathfrak{g}) \rightarrow \operatorname{Inn} A u t(\mathcal{G})$, where $G(\mathfrak{g})$ is the 1-connected Lie group integrating $\mathfrak{g}$.

Proof. By the assumptions, we have a Lie group action $G(\mathfrak{g}) \times M \rightarrow M$, and the corresponding action groupoid $G(\mathfrak{g}) \ltimes M \rightrightarrows M$ is source 1-connected. Furthermore, the Lie algebroid morphism $\psi: \mathfrak{g} \ltimes M \rightarrow A$ integrates to a Lie groupoid morphism $\tilde{\Psi}: G(\mathfrak{g}) \ltimes M \rightarrow \mathcal{G}(A)$. Denote by $\Psi$ the composition of $\tilde{\Psi}$ with the natural projection $\mathcal{G}(A) \rightarrow \mathcal{G}$. Then, one obtains an inner action $\boldsymbol{A}: G(\mathfrak{g}) \rightarrow \operatorname{Inn} \operatorname{Aut}(\mathcal{G})$ by (29). As is easily checked, it integrates the inner action $\mathfrak{a}_{*}: \mathfrak{g} \rightarrow \operatorname{InnDer}(A)$.

The above result is slightly better than the integration of non-inner actions we referred to in the end of the preceding subsection. In general, in order to integrate a Lie algebra action of $\mathfrak{g}$ on a Lie algebroid $A$ to a Lie group action of $G$ on a Lie groupoid $\mathcal{G}$, we need both $G$ to be 1 -connected and $\mathcal{G}$ to be source 1-connected (and the action to be complete).

This is important for our purposes, as $G$ is the structure group of a principal bundle, thus its topology is imposed. So, we need to refine Proposition A.17 to groups that are neither simply connected nor connected.

Hence, assume that we want to integrate an inner action $\mathfrak{a}: \mathfrak{g} \rightarrow \operatorname{InnDer}(A)$ associated with $\psi: \mathfrak{g} \ltimes M \rightarrow A$ to an action of a connected (but not necessarily 
1-connected) Lie group $G$ with Lie algebra $\mathfrak{g}$. Recall that for a connected Lie group $G$, its fundamental group fits into the exact sequence

$$
1 \longrightarrow \pi_{1}(G) \longrightarrow G(\mathfrak{g}) \longrightarrow G \longrightarrow 1 .
$$

Proposition A.18. Suppose that $G$ is a connected Lie group acting on a manifold $M, \mathcal{G}$ is a Lie groupoid over $M$, and $\bar{\Psi}: G(\mathfrak{g}) \ltimes M \rightarrow \mathcal{G}$ is a Lie groupoid morphism. Then, $\bar{\Psi}$ descends to a Lie groupoid morphism $\Psi: G \ltimes M \rightarrow \mathcal{G}$ if and only if it takes values in units when restricted to $\pi_{1}(G) \ltimes M \subset G(\mathfrak{g}) \ltimes M$, namely

$$
\bar{\Psi}\left(\pi_{1}(G) \ltimes M\right)=\mathbf{1}_{M} .
$$

The proof is left to the reader. Note that although $\psi: \mathfrak{g} \ltimes M \rightarrow A$ may not integrate to $\Psi: G \ltimes M \rightarrow \mathcal{G}$, it may still integrate to a morphism $\Psi^{\prime}: G \ltimes M \rightarrow \mathcal{G}^{\prime}$ for a smaller Lie groupoid $\mathcal{G}^{\prime}$ integrating $A$. This can be decided as follows. First we integrate $\psi: \mathfrak{g} \ltimes M \rightarrow A$ to a Lie groupoid morphism $\tilde{\Psi}: G(\mathfrak{g}) \ltimes M \rightarrow \mathcal{G}(A)$ with values in the source 1-connected Lie groupoid integrating $A$. Then, given any connected Lie group $G$ integrating $\mathfrak{g}$, we introduce a bundle of groups $\Delta$ over $M$, defined in the following way:

$$
\Delta:=\tilde{\Psi}\left(\pi_{1}(G) \ltimes M\right) \subset \mathcal{G}(A)
$$

Recall that if $\Delta$ is a totally disconnected wide normal subgroupoid of $\mathcal{G}(A)$, then the quotient $\mathcal{G}(A) / \Delta$ is a Lie groupoid; see, e.g., the discussion in [Gualtieri and Li 2014, Theorem 1.14]. Furthermore, it is easy to see that $\mathcal{G}(A) / \Delta$ integrates $A$. Therefore, we obtain a Lie groupoid morphism:

$$
\Psi: G \ltimes M \rightarrow \mathcal{G}(A) / \Delta .
$$

Of course, $\psi$ integrates to a morphism $G \ltimes M \rightarrow \mathcal{G}$ whenever $\mathcal{G}$ is covered by $\mathcal{G}_{0}:=\mathcal{G}(A) / \Delta$.

Theorem A.19. Let $\boldsymbol{a}: G \rightarrow \operatorname{Aut}(A)$ be a prehamiltonian action of a Lie group $G$ on a Lie algebroid $A$ with premoment map $\psi_{*}: \mathfrak{g} \rightarrow \Gamma(A)$ and:

- $\psi: \mathfrak{g} \ltimes M \rightarrow A$ the Lie algebroid associated with $\psi_{*}$,

- $\tilde{\Psi}: G(\mathfrak{g}) \ltimes M \rightarrow \mathcal{G}(A)$ the groupoid morphism integrating $\psi$,

- $\Delta \subset \mathcal{G}(A)$, the subset defined by $\Delta:=\tilde{\Psi}\left(\pi_{1}(G) \ltimes M\right) \subset \mathcal{G}(A)$.

Then, the following assertions hold:

(i) $\Delta$ is a wide, normal, totally disconnected subgroupoid of $\mathcal{G}(A)$,

(ii) a integrates to a groupoid action $\boldsymbol{A}: G \rightarrow \operatorname{Aut}(\mathcal{G}(A) / \Delta)$. 
Moreover, $\mathcal{G}(A) / \Delta$ is a Lie groupoid if and only if $\Delta \subset \mathcal{G}(A)$ is an embedding. In this case, $\tilde{\Psi}$ descends to a Lie groupoid morphism $\Psi: G \times M \rightarrow \mathcal{G}(A) / \Delta$ and

$$
\begin{aligned}
\boldsymbol{A}_{h}(x) & =\Psi_{n}(h) \cdot x \cdot \Psi_{m}(h)^{-1} \\
\Psi_{g m}\left(g h g^{-1}\right) & =A_{g}\left(\Psi_{m}(h)\right)
\end{aligned}
$$

for any $h \in G^{\circ}, x \in \mathcal{G}(A) / \Delta, g \in G$, where $m:=\boldsymbol{s}(x)$ and $n:=\boldsymbol{t}(x)$.

Proof. Since $G$ acts on $A$ by Lie algebroid automorphisms, one can lift $\boldsymbol{a}$ into an action $\tilde{\boldsymbol{A}}$ of $G$ on $\mathcal{G}(A)$ by groupoid automorphisms via

$$
\tilde{\boldsymbol{A}}_{g}\left([q]_{A}\right):=\left[\boldsymbol{a}_{g} \circ q\right]_{A}, \quad \text { for } q \in P(A), g \in G .
$$

Consider now an $A$-path $q$ and an element $h$ lying in the neutral component $G^{\circ}$ of G. We extend $q$ into a time dependent section of $A$ (which we still denote by $q$ ) and consider any $\mathfrak{g}$-path $\xi: \epsilon \mapsto \xi_{\epsilon} \in \mathfrak{g}$ that induces a path $h_{\epsilon}$ in $G^{\circ}$ between the identity and $h$. By the construction, $\left(\boldsymbol{a}_{h_{\epsilon}}\right)_{*}\left(q_{t}\right)$ is a solution of the evolution equation

$$
\left[\left(\boldsymbol{a}_{h_{\epsilon}}\right)_{*}\left(q_{t}\right), \psi_{*}\left(\xi_{\epsilon}\right)\right]_{A}=\frac{\mathrm{d}}{\mathrm{d} \epsilon}\left(\boldsymbol{a}_{h_{\epsilon}}\right)_{*}\left(q_{t}\right), \quad \text { for } \epsilon, t \in I .
$$

Then, by [Brahic 2010, Proposition A.1], we obtain

$$
\tilde{\boldsymbol{A}}_{h}\left([q]_{A}\right)=\tilde{\Psi}_{y}\left([\xi]_{\mathfrak{g}}\right) \cdot[q]_{A} \cdot \tilde{\Psi}_{x}\left([\xi]_{\mathfrak{g}}\right)^{-1} .
$$

In particular, if $h=1$, that is, $\xi_{\epsilon}$ induces a loop in $G^{\circ}$, then $\Delta$ is a normal subgroupoid of $\mathcal{G}(A)$.

Next, we have to make sure that $\tilde{\boldsymbol{A}}$ induces an action on $\mathcal{G}(A) / \Delta$, so we need to check that $\tilde{A}_{h}(\Delta)=\Delta$. For this, we apply Equation (34) with $q=\tilde{\Psi}[\eta]_{\mathfrak{g}}$, where $\eta$ is a $\mathfrak{g}$-path inducing a loop in $G$, and we use successively the fact that $\tilde{\Psi}$ is a Lie groupoid homomorphism, then that $\pi_{1}(G)$ lies in $G(\mathfrak{g})$ as a normal subgroup. The first relation follows. The second one is obtained by using the equivariance condition in Definition A.16.

Example A.20. Here is a basic example where the resulting groupoid $\mathcal{G}(A) / \Delta$ is not smooth. Consider the 1-dimensional (abelian) Lie algebra $\mathfrak{z}=\mathbb{R}$, its dual $\mathfrak{z}^{*}$ endowed with the trivial linear Poisson structure, and $A:=T^{*} \mathfrak{z}^{*} \simeq \mathfrak{z} \ltimes \mathfrak{z}^{*}$ the corresponding Lie algebroid. Then, $A$ integrates to a bundle of Lie groups $Z \ltimes \mathfrak{z}^{*}$, where $Z$ is the Lie group $\mathbb{R}$.

Consider furthermore the trivial action of $G:=S^{1} \simeq \mathbb{R} / 2 \pi \mathbb{Z}$ on $\mathfrak{z}^{*}$. Then, any application $J: \mathfrak{z}^{*} \rightarrow \operatorname{Lie}\left(S^{1}\right)$ can be chosen to be a moment map. From the Lie algebroid point of view (see Definition A.16) we only have a prehamiltonian moment map $\psi_{*}: \operatorname{Lie}\left(S^{1}\right) \rightarrow \Gamma\left(\mathfrak{z} \ltimes \mathfrak{z}^{*}\right), X \mapsto \mathrm{d} J(X)$. The corresponding Lie algebroid morphism $\psi: \operatorname{Lie}\left(S^{1}\right) \ltimes \mathfrak{z}^{*} \rightarrow \mathfrak{z} \ltimes \mathfrak{z}^{*}$ is given by $(X, z) \mapsto\left(\mathrm{d} J_{z}(X), z\right)$. It integrates 
to a Lie groupoid morphism $\mathbb{R} \ltimes \mathfrak{z}^{*} \rightarrow Z \ltimes \mathfrak{z}^{*}$ given by $(\theta, z) \mapsto\left(\mathrm{d} J_{z}(\theta), z\right)$. The exact sequence (31) reads

$$
1 \longrightarrow 2 \longrightarrow \mathbb{R} \longrightarrow S^{1} \longrightarrow 1
$$

Hence, we obtain $\Delta_{z}=\left\{\left(\mathrm{d} J_{z}(2 k \pi), z\right): k \in \mathbb{Z}\right\}$. Clearly, $\Delta$ defines a normal subgroupoid of $Z \ltimes \mathfrak{z}^{*}$; however, $Z \ltimes \mathfrak{z}^{*} / \Delta$ is not smooth if $\mathrm{d} J$ vanishes at some point $z_{0}$.

\section{References}

[Brahic 2010] O. Brahic, "Extensions of Lie brackets", J. Geom. Phys. 60:2 (2010), 352-374. MR 2011b:58038 Zbl 1207.58018

[Brahic and Fernandes 2008] O. Brahic and R. L. Fernandes, "Poisson fibrations and fibered symplectic groupoids", pp. 41-59 in Proceedings of the 5th International Conference (Tokyo, 2006), edited by G. Dito et al., Contemp. Math. 450, Amer. Math. Soc., Providence, RI, 2008. MR 2009h:53185 Zbl 1158.53064

[Brahic and Fernandes 2014] O. Brahic and R. L. Fernandes, "Integrability and reduction of Hamiltonian actions on Dirac manifolds", Indag. Math. (N.S.) 25:5 (2014), 901-925. MR 3264780 Zbl 1298.53082

[Brahic and Zhu 2011] O. Brahic and C. Zhu, "Lie algebroid fibrations", Adv. Math. 226:4 (2011), 3105-3135. MR 2012a:53156 Zbl 1210.53076

[Bursztyn et al. 2004] H. Bursztyn, M. Crainic, A. Weinstein, and C. Zhu, "Integration of twisted Dirac brackets”, Duke Math. J. 123:3 (2004), 549-607. MR 2005e:53128 Zbl 1067.58016

[Courant 1990] T. J. Courant, "Dirac manifolds", Trans. Amer. Math. Soc. 319:2 (1990), 631-661. MR 90m:58065 Zbl 0850.70212

[Crainic 2004] M. Crainic, "Prequantization and Lie brackets", J. Symplectic Geom. 2:4 (2004), 579-602. MR 2006j:53129 Zbl 1095.53060

[Crainic and Fernandes 2003] M. Crainic and R. L. Fernandes, "Integrability of Lie brackets", Ann. of Math. (2) 157:2 (2003), 575-620. MR 2004h:58027 Zbl 1037.22003

[Crainic and Fernandes 2004] M. Crainic and R. L. Fernandes, "Integrability of Poisson brackets", J. Differential Geom. 66:1 (2004), 71-137. MR 2006f:53119 Zbl 1066.53131

[Crainic and Fernandes 2011] M. Crainic and R. L. Fernandes, "Lectures on integrability of Lie brackets", pp. 1-107 in Lectures on Poisson geometry (Trieste, 2005), edited by T. Ratiu et al., Geom. Topol. Monogr. 17, 2011. MR 2795150 Zbl 1227.22005

[Dufour and Wade 2008] J.-P. Dufour and A. Wade, "On the local structure of Dirac manifolds", Compos. Math. 144:3 (2008), 774-786. MR 2010c:53121 Zbl 1142.53063

[Fernandes et al. 2009] R. L. Fernandes, J.-P. Ortega, and T. S. Ratiu, "The momentum map in Poisson geometry”, Amer. J. Math. 131:5 (2009), 1261-1310. MR 2011f:53199 Zbl 1180.53083

[Gualtieri and Li 2014] M. Gualtieri and S. Li, "Symplectic groupoids of log symplectic manifolds", Int. Math. Res. Not. 2014:11 (2014), 3022-3074. MR 3214314 Zbl 1305.22004

[Guillemin et al. 1996] V. Guillemin, E. Lerman, and S. Sternberg, Symplectic fibrations and multiplicity diagrams, Cambridge University Press, Cambridge, 1996. MR 98d:58074 Zbl 0870.58023

[Mărcuţ and Frejlich 2013] I. Mărcuţ and P. Frejlich, "The normal form theorem around Poisson transversals", preprint, 2013. arXiv 1306.6055v2 
[McDuff and Salamon 1998] D. McDuff and D. Salamon, Introduction to symplectic topology, 2nd ed., Clarendon Press, New York, 1998. MR 2000g:53098

[Vaisman 2006] I. Vaisman, "Foliation-coupling Dirac structures", J. Geom. Phys. 56:6 (2006), 917-938. MR 2007f:53109 Zbl 1109.53076

[Vorobjev 2001] Y. Vorobjev, "Coupling tensors and Poisson geometry near a single symplectic leaf", pp. 249-274 in Lie algebroids and related topics in differential geometry (Warsaw, 2000) (Warsaw, 2000), edited by J. Kubarski and R. Urbański, Pawełand Wolak, Banach Center Publ. 54, Polish Acad. Sci. Inst. Math., Warsaw, 2001. MR 2003d:53145 Zbl 1007.53062

[Wade 2008] A. Wade, "Poisson fiber bundles and coupling Dirac structures", Ann. Global Anal. Geom. 33:3 (2008), 207-217. MR 2009d:53122 Zbl 1151.53070

[Weinstein 1980] A. Weinstein, "Fat bundles and symplectic manifolds", Adv. in Math. 37:3 (1980), 239-250. MR 82a:53038 Zbl 0449.53035

Received October 6, 2014. Revised March 26, 2015.

OLIVIER BRAHIC

DEPARTMENT OF MATHEMATICS

FEDERAL UNIVERSITY OF PARANÁ

CP 19081

81531-980 CURITIBA-PR

BRAZIL

brahicolivier@gmail.com

RUI LOJA FERNANDES

DEPARTMENT OF MATHEMATICS

UNIVERSITY OF ILLINOIS AT URBANA-CHAMPAIGN

1409 W. GREEN STREET

URBANA, IL 61801

UNITED STATES

ruiloja@illinois.edu 


\title{
PACIFIC JOURNAL OF MATHEMATICS
}

\author{
msp.org/pjm
}

Founded in 1951 by E. F. Beckenbach (1906-1982) and F. Wolf (1904-1989)

\section{EDITORS}

Don Blasius (Managing Editor)

Department of Mathematics

University of California

Los Angeles, CA 90095-1555

blasius@math.ucla.edu

\author{
Paul Balmer \\ Department of Mathematics \\ University of California \\ Los Angeles, CA 90095-1555 \\ balmer@math.ucla.edu \\ Robert Finn \\ Department of Mathematics \\ Stanford University \\ Stanford, CA 94305-2125 \\ finn@math.stanford.edu \\ Sorin Popa \\ Department of Mathematics \\ University of California \\ Los Angeles, CA 90095-1555 \\ popa@math.ucla.edu
}

\author{
Vyjayanthi Chari \\ Department of Mathematics \\ University of California \\ Riverside, CA 92521-0135 \\ chari@math.ucr.edu \\ Kefeng Liu \\ Department of Mathematics \\ University of California \\ Los Angeles, CA 90095-1555 \\ liu@math.ucla.edu \\ Jie Qing \\ Department of Mathematics \\ University of California \\ Santa Cruz, CA 95064 \\ qing@ cats.ucsc.edu
}

\section{PRODUCTION}

Silvio Levy, Scientific Editor, production@msp.org

\section{SUPPORTING INSTITUTIONS}

ACADEMIA SINICA, TAIPEI

CALIFORNIA INST. OF TECHNOLOGY

INST. DE MATEMÁTICA PURA E APLICADA

KEIO UNIVERSITY

MATH. SCIENCES RESEARCH INSTITUTE

NEW MEXICO STATE UNIV.

OREGON STATE UNIV.

\author{
STANFORD UNIVERSITY \\ UNIV. OF BRITISH COLUMBIA \\ UNIV. OF CALIFORNIA, BERKELEY \\ UNIV. OF CALIFORNIA, DAVIS \\ UNIV. OF CALIFORNIA, LOS ANGELES \\ UNIV. OF CALIFORNIA, RIVERSIDE \\ UNIV. OF CALIFORNIA, SAN DIEGO \\ UNIV. OF CALIF., SANTA BARBARA
}

\author{
Daryl Cooper \\ Department of Mathematics \\ University of California \\ Santa Barbara, CA 93106-3080 \\ cooper@math.ucsb.edu \\ Jiang-Hua Lu \\ Department of Mathematics \\ The University of Hong Kong \\ Pokfulam Rd., Hong Kong \\ jhlu@maths.hku.hk \\ Paul Yang \\ Department of Mathematics \\ Princeton University \\ Princeton NJ 08544-1000 \\ yang@math.princeton.edu
}

These supporting institutions contribute to the cost of publication of this Journal, but they are not owners or publishers and have no responsibility for its contents or policies.

See inside back cover or msp.org/pjm for submission instructions.

The subscription price for 2015 is US \$420/year for the electronic version, and \$570/year for print and electronic.

Subscriptions, requests for back issues and changes of subscribers address should be sent to Pacific Journal of Mathematics, P.O. Box 4163, Berkeley, CA 94704-0163, U.S.A. The Pacific Journal of Mathematics is indexed by Mathematical Reviews, Zentralblatt MATH, PASCAL CNRS Index, Referativnyi Zhurnal, Current Mathematical Publications and Web of Knowledge (Science Citation Index).

The Pacific Journal of Mathematics (ISSN 0030-8730) at the University of California, c/o Department of Mathematics, 798 Evans Hall \#3840, Berkeley, CA 94720-3840, is published twelve times a year. Periodical rate postage paid at Berkeley, CA 94704, and additional mailing offices. POSTMASTER: send address changes to Pacific Journal of Mathematics, P.O. Box 4163, Berkeley, CA 94704-0163.

PJM peer review and production are managed by EditFLOW ${ }^{\circledR}$ from Mathematical Sciences Publishers.

\section{PUBLISHED BY}

\section{mathematical sciences publishers \\ nonprofit scientific publishing}

http://msp.org/

(C) 2015 Mathematical Sciences Publishers 


\section{PACIFIC JOURNAL OF MATHEMATICS}

Volume $278 \quad$ No. $2 \quad$ December 2015

Differential Harnack and logarithmic Sobolev inequalities along

Ricci-harmonic map flow

ABIMBOLA ABOLARINWA

On $J$-holomorphic curves in almost complex manifolds with

291

asymptotically cylindrical ends

ERKAO BAO

Integration of coupling Dirac structures

OLIVIER BRAHIC and RUI LOJA FERNANDES

Asymptotic behavior of Palais-Smale sequences associated with fractional Yamabe-type equations

Yi FANG and MARÍA DEL MAR GONZÁLEZ

$K$-theory and homotopies of 2-cocycles on higher-rank graphs

ELIZABETH GILLASPY

Fusion products and toroidal algebras

DENIZ KUS and PETER LiTTELMANN

Differential Harnack estimates for positive solutions to heat equation under Finsler-Ricci flow

\section{SAJJAD LAKZIAN}

On the one-endedness of graphs of groups

NichOLAS TOUIKAN

On the structure of vertex cuts separating the ends of a graph

GARETH R. WILKES 